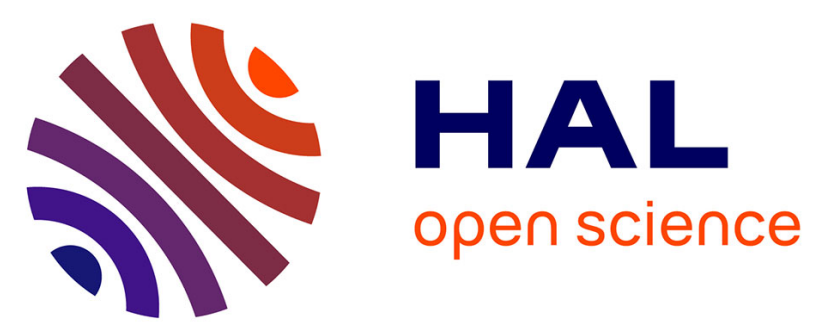

\title{
ÉTUDES SUR LES VARIATIONS DE LA RICHESSE EN CONSTITUANTS AZOTÉS DES LAITS DE VACHE. IV. - RELATION ENTRE LA TENEUR EN PROTEINES ET LE TAUX BUTYREUX
}

\author{
C. Rossetti, R. Jarrige
}

\section{To cite this version:}

C. Rossetti, R. Jarrige. ÉTUDES SUR LES VARIATIONS DE LA RICHESSE EN CONSTITUANTS AZOTÉS DES LAITS DE VACHE. IV. - RELATION ENTRE LA TENEUR EN PROTEINES ET LE TAUX BUTYREUX. Annales de zootechnie, 1957, 6 (2), pp.121-150. hal-00886669

\section{HAL Id: hal-00886669 https://hal.science/hal-00886669}

Submitted on 1 Jan 1957

HAL is a multi-disciplinary open access archive for the deposit and dissemination of scientific research documents, whether they are published or not. The documents may come from teaching and research institutions in France or abroad, or from public or private research centers.
L'archive ouverte pluridisciplinaire HAL, est destinée au dépôt et à la diffusion de documents scientifiques de niveau recherche, publiés ou non, émanant des établissements d'enseignement et de recherche français ou étrangers, des laboratoires publics ou privés. 


\section{ÉTUDES SUR LES VARIATIONS DE LA RICHESSE EN CONSTITUANTS AZOTÉS DES LAITS DE VACHE}

\section{IV. - RELATION ENTRE LA TENEUR EN PROTEINES ET LE TAUX BUTYREUX}

\section{ROSSETTI et R. JARRIGE (i)}

PAR

Station de recherches sur 1'Élevage, C. N. R. Z., Jouy-en-Josas.

Nous avons montré précédemment (JARRIGE et ROSSETTI, I957 $b$ ) que la teneur moyenne en constituants azotés présente, entre vaches de la même race, des différences sensibles et reproductibles qui seraient largement héritables (HaNCOCK I953). Il est donc possible de tenir compte de ce caractère dans la sélection du bétail laitier, ce qui pose deux problèmes pratiques par rapport à la méthode classique de sélection sur le taux butyreux :

I $^{0}$ Celle-ci permet-elle une amélioration efficace de la richesse en protéines? S'il en est ainsi, il est inutile de déterminer directement la teneur en azote qu'on pourra estimer à partir du taux butyreux ;

$2^{\circ}$ Peut-on augmenter indépendamment la teneur en protéines et le taux butyreux, de façon à obtenir d'une part des laits "beurriers " riches en matières grasses et relativement pauvres en azote (LoNkA I947), et d'autre part, des laits "fromagers " avant tout riches en caséine.

Ces problèmes nous amènent à envisager les variations de la teneur en protéines, sous les aspects théoriques suivants :

Io Une partie de ces variations est-elle liée à celles du taux butyreux?

$2^{\circ}$ Quelle est l'importance de cette liaison et corrélativement, celle de la variation indépendante?

$3^{\circ}$ Quelles sont les causes qui déterminent cette liaison ; celle-ci présente-t-elle des variations de nature et d'intensité entre vaches de la même race?

(1) Avec la collaboration technique de $\mathrm{I}^{\mathrm{me}} \mathrm{J}$. Juxg, Ime C. Le Gallo et R. DCPIN. 


\section{'Tableau I}

Relations entre les teneurs en matières azotées $(y)$ et le taux butyreux $(x)$ des laits individuels et des laits de mélange.

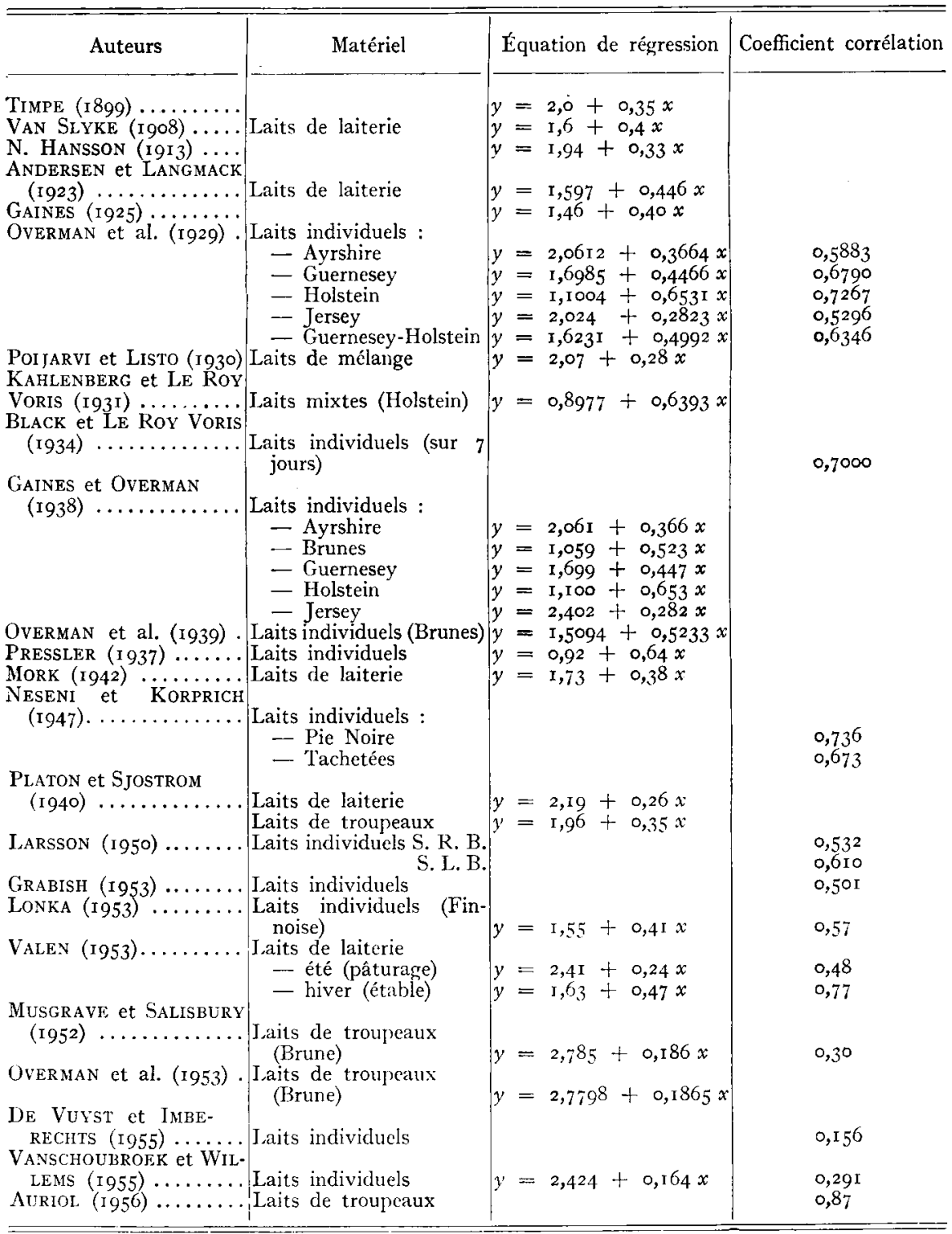

I.es nombreux travaux sur les laits de mélange et les laits individuels résumés au tableau. I ont établi qu'il existe une liaison positive plus ou moins étroite entre teneur en protéines et taux butyreux ; ils n'apportent 


\section{TABLEAU II}

Relations inter-individuelles entre les teneurs pondérées en matières azotées $(y)$ et matières grasses $(x)$.

\begin{tabular}{|c|c|c|c|}
\hline Auteurs & Matériel & Équation de régression & $\begin{array}{c}\text { Coefficient } \\
\text { de corrélation }\end{array}$ \\
\hline 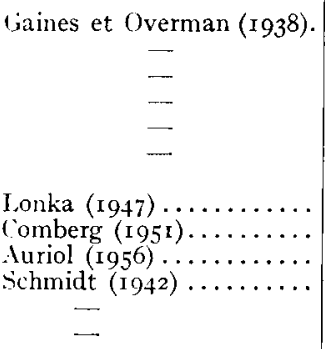 & $\begin{array}{c}\text { Ayrshire (1 4) } \\
\text { Brune des Alpes (I } 7) \\
\text { Guernesey (I } 4) \\
\text { Holstein (I 5) } \\
\text { Jersey (I 3) } \\
\text { Ensemble des } \\
\text { I 30 vaches } \\
\text { Finoises (54) } \\
\text { Schwarzbunte (42) } \\
\text { Pie Rouge de l'Est (235) } \\
\text { Schwarzbunte (10) } \\
\text { Rouge Danoise (10) } \\
\text { Jersey (I0) }\end{array}$ & $\begin{array}{l}y=2,257+0,298 x \\
y=2,091+0,350 x \\
y=0,860+0,594 x \\
y=1,514+0,527 x \\
y=1,590+0,43^{8} x \\
y=2,100+0,346 x \\
y=1,53+0,39 x \\
y=21,83+0,282 x \\
y=1,95+0,356 x\end{array}$ & $\begin{array}{l}0,674 \\
0,48\end{array}$ \\
\hline
\end{tabular}

cependant aucune réponse aux deux autres problèmes, et surtout ne permettent pas de conclure, comme on 1'a fait généralement et implicitement que la sélection sur le taux butyreux entraîne une amélioration simultanée de la teneur en protéines. KRIZENECKY (I943) les a discutés et a montré qu'il fallait attacher beaucoup plus d'importance aux liaisons intra-individuelles (ensemble des laits d'une même vache) et surtout inter-individuelles (chaque vache étant représentée par ses valeurs moyennes). Ces dernières catégories de calculs (tableau II) [GAINEs et OVERMan (I938), Pressler (I937), Lonka (I947), Piel (I953), Comberg (I95I), AUriol (I956)], jointes à la méthode plus récente d'analyse hiérarchique de la variance [BONNIER et HANsson (I946), HANsson et BONNIER (I950), LARsson et a1. (I949)] ont mis en évidence les différences individuelles de nature vraisemblablement héréditaire, dans les relations entre taux butyreux et teneur en protéines.

Sur le matériel limité que nous avons présenté précédemment (cf. JARRIGE et ROSSETTI, I957 a) nous allons reprendre ces diverses méthodes suivant leur ordre chronologique pour :

- démontrer l'existence de relations entre ces deux constituants, aux différents niveaux (ensemble des laits, intra-vaches, inter-vaches),

- montrer qu'elles sont variables d'un animal à l'autre,

— et surtout, analyser leur signification et dégager leurs conséquences.

Rappelons que nous avons suivi 28 vaches (tableau III) au cours d'une lactation (réalisée dans des conditions comparables), à l'aide d'en moyenne 30 échantillons constitués sur $4^{8}$ heures et répartis de la façon 
suivante : un par semaine au cours des deux premiers et des deux derniers mois de lactation et un par quinzaine dans l'intervalle.

\section{Ensemble des échantillons individuels}

\section{Existence d'une liaison : corrélation et régression linéaires.}

La première méthode utilisée pour mettre en évidence une liaison entre la teneur en protéines a été de reporter sur un tableau de corrélation, l'ensemble des analyses de laits de mélange ou de laits individuels, indépendamment de leur provenance (vache-stade lactation...), et de calculer une droite de régression.

Cette méthođe, appliquée à l'ensemble de nos 855 échantillons, nous révèle une liaison à ce niveau avec des valeurs tout à fait comparables aux résultats antérieurs réunis dans le tableau $\mathrm{I}$.

$$
\begin{aligned}
y & =\mathrm{I} 7,68+0,44 \mathrm{I} x \\
r_{x y} & =0,675^{\times \times} \mp \text { o,org. }
\end{aligned}
$$

Il faut immédiatement remarquer que, comme les autres auteurs, nous avons fait une adaptation à une droite sans avoir démontré que la liaison était linéaire. Ce fait a été admis implicitement par la plupart des auteurs du tableau I, et confirmé par l'étude critique de KRIZENECKY (I943) ; par contre, les auteurs scandinaves ont montré que cette liaison est curvilinéaire à l'échelle d'un ensemble de laits individuels, la teneur en protéines augmentant plus vite pour les taux butyreux plus élevés (Bonnier et Hansson I946, Larsson I950). Comme le montre le graphique 2 où nous avons reporté les moyennes de la teneur en protéines par classe de taux butyreux ( $\mathrm{I}$ point), il en est peu être de même dans notre ensemble de laits : nous n'avons cependant pas recherché l'équation de cette régression curvilinéaire, car ce calcul serait purement formel et stérile, notre matériel étant hétérogène.

Pour mettre en évidence cette hétérogénéité, nous avons calculé les mêmes régressions linéaires pour l'ensemble des laits de chacune des races et obtenu les valeurs suivantes :

$$
\begin{aligned}
& \text { vaches frisonnes } 49 \mathrm{I} \text { échantillons } \quad y=27,9 \mathrm{I}+0, \mathbf{1} 32 x \\
& r_{x y}=0, \mathrm{I} 54^{\times \times} \mp 0,044 \\
& \text { vaches normandes } 334 \text { échantillons } \quad y=34,2 \mathrm{I}+0,0674 x \\
& r_{x y}=0,090 \pm 0,055 \text {. }
\end{aligned}
$$

I,e coefficient de corrélation est, cette fois-ci, beaucoup plus faible (très significativement inférieur au précédent) : il est cependant significatif pour les vaches Frisonnes, mais non pour les Normandes. Nous ne retrouvons plus, à l'échelle de la race, qu'une liaison très faible alors qu'elle était très forte pour l'ensemble des laits. Le graphique I nous explique immédiatement ce paradoxe : les laits des Normandes étant plus riches 


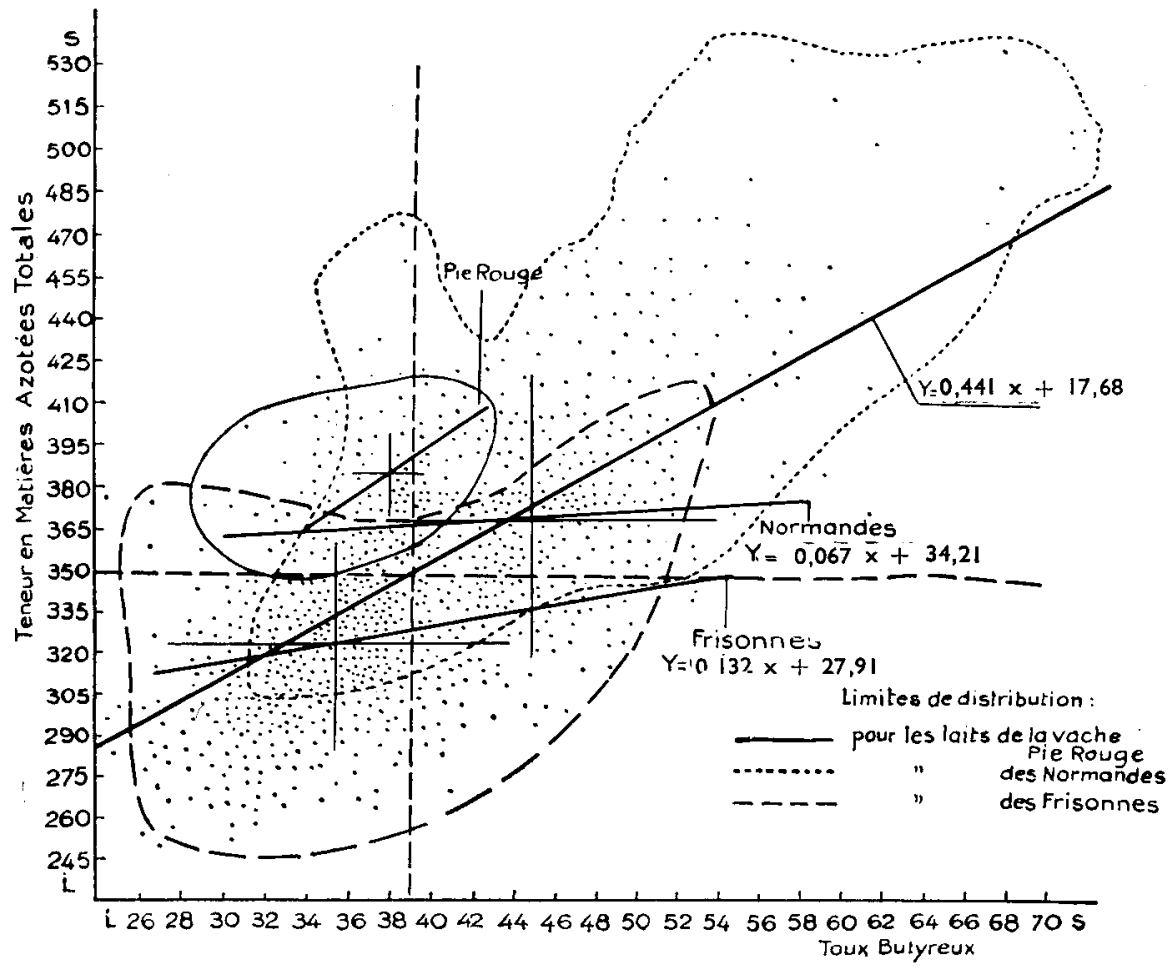

GRAPHIQUE I. - Corrélation entre la teneur en matières azotées et le taux butyreux des laits individuels.

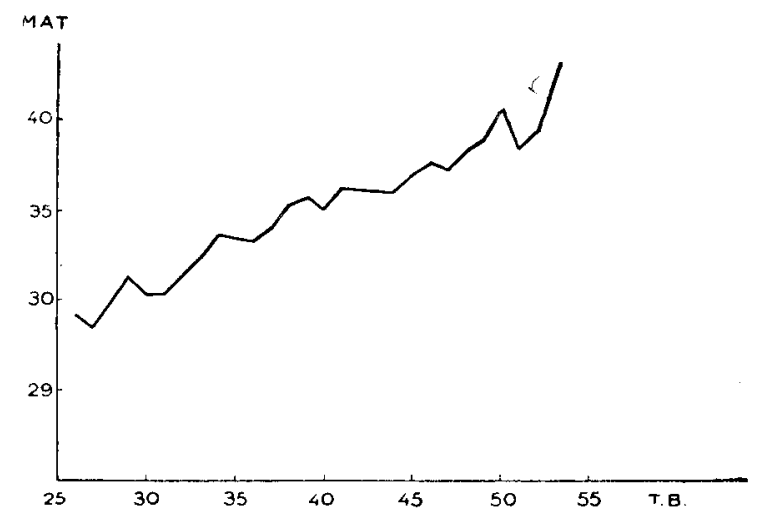

GraphiǫeE 2. - Ensemble des laits individuels : teneur moyenne en matières azotées totales par classe de taux butyreux (intervalle : I gramme).

que ceux des Frisonnes en matières grasses et en matières azotées, nous avons deux populations distinctes : si on les réunit, on obtient un nuage de points ellipsoïdal dont le grand axe a une pente élevée ; pour parfaire 
cette ellipse, les laits de l'unique vache pie rouge viennent combler un vide dans le quadrant supérieur gauche.

Ainsi, nous avons bien une corrélation positive entre teneur en protéines et taux butyreux, mais sa valeur traduit avant tout les artifices de l'échantillonnage.Un problème surgit immédiatement : pourquoi ne retrouvons-nous qu'une liaison très faible pour l'ensemble des laits de chaque race? Toujours pour la même raison : cet ensemble est hétérogène et correspond à la juxtaposition plus ou moins nette des populations individuelles groupant chacune les valeurs d'une même vache. A 1'intérieur de chacune d'elles, existe en moyenne une liaison fondamentale (tableau III), qui disparaît si on groupe les vaches de la même race, mais renait si on réunit les vaches des deux races. Ce « petit jeu de cachecache " illustre fort bien la stérilité de ces calculs statistiques appliqués sans discernement à des ensembles hétérogènes de laits individuels et, à plus forte raison, de laits de mélange ; ils révèlent des tendances, mais ne permettent aucune conclusion génétique. Pour étayer cette critique, il nous faut maintenant démontrer l'existence de ces populations individuelles grâce à l'analyse hiérarchique de la variance.

\section{Analyse de la variance.}

L'analyse des relations entre le taux butyreux et la teneur en protéines est d'abord perturbée par le fait que ces deux constituants évoluent de façon sensiblement parallèle au cours de la lactation (cf. JARRIGE et RossETtr I957 a). Pour éliminer cette interférence, nous avons donc d'abord classé les échantillons par stade de lactation en 6 périodes successives de deux mois : sauf dans la première et la dernière, on peut admettre que la composition du lait demeure sensiblement constante dans chacune d'elles. A l'intérieur de chacune de ces périodes, nous avons réparti les échantillons en I5 classes de taux butyreux : $<25 ; 25$ à $27 ; 27$ à $29 \ldots$; nous avons dû prendre cet intervalle de 2 points par suite du nombre élevé de variables vis-à-vis du nombre limité d'échantillons ; nous pouvons admettre cependant que la variation du taux butyreux à l'intérieur de ces classes est suffisamment faible pour qu'on puisse négliger les variations de la teneur en protéines qui lui sont liées. Nous avons ensuite séparé les échantillons des Normandes et des Frisonnes et calculé : à l'intérieur de ces nouvelles classes les variations inter-vaches et intra vaches (tableau IV).

Après avoir éliminé l'interférence prépondérante du stade de lactation, cette analyse nous montre des influences significatives hiérarchisées du taux butyreux, de la race et de l'individu, et permet de dégager les faits suivants :

$\mathrm{I}^{0}$ I,es teneurs en matières azotées correspondant aux diverses classes 


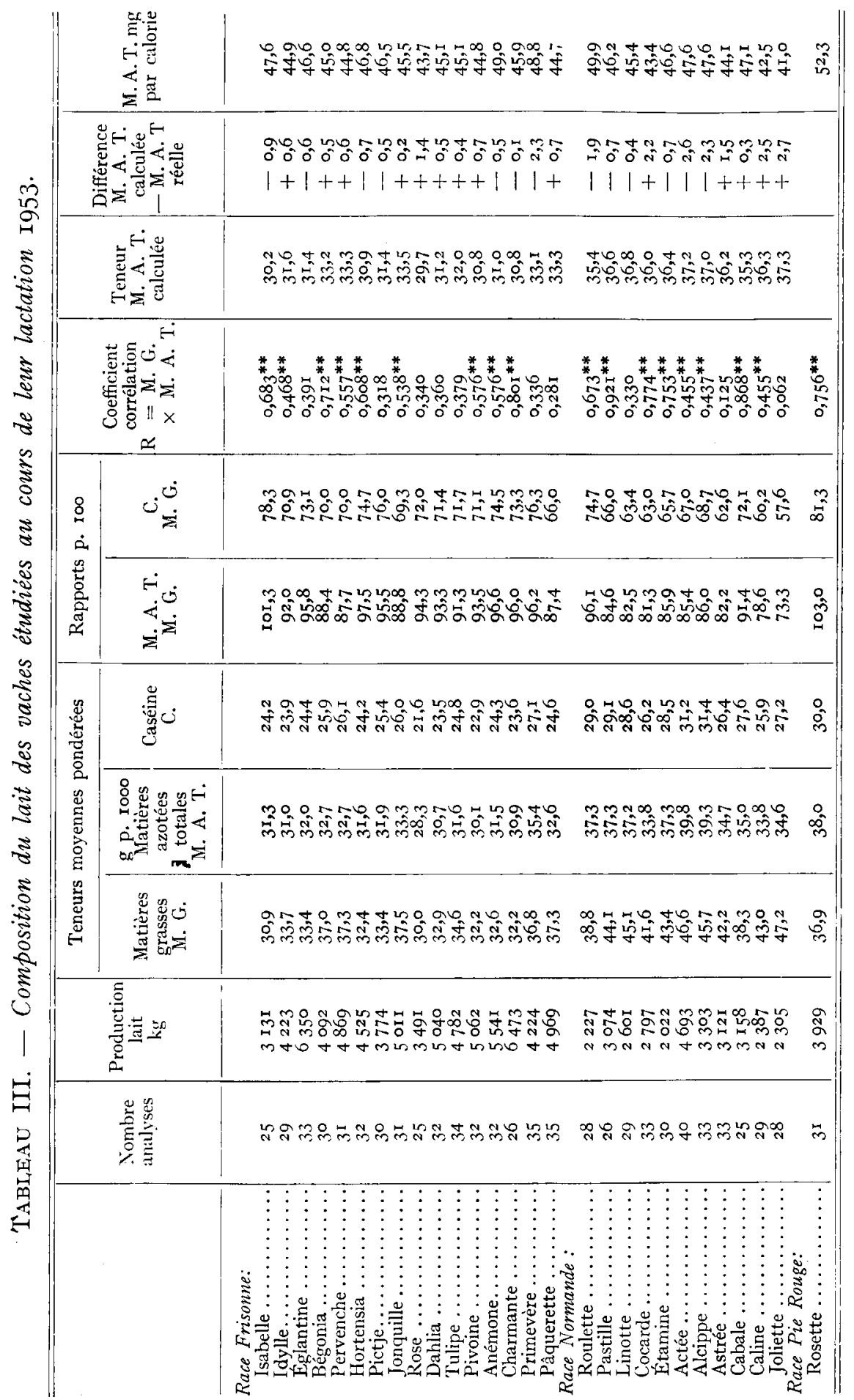


TABLEAU IV

Analyse de la variance de la teneur en matières azotées totales.

\begin{tabular}{|c|c|c|c|c|}
\hline Causes de variation & $\begin{array}{l}\text { Somme } \\
\text { des carrés }\end{array}$ & $\begin{array}{l}\text { Degrés } \\
\text { de liberté }\end{array}$ & Variance & Rapport \\
\hline Total............. & 18210,708 & 831 & & \\
\hline Entre stades de lactation......... & 5722,928 & 5 & I I I 4,580 & $75,7 \circ 9^{\times x}$ \\
\hline A l'intérieur des stades de lactation & I 2487,780 & 826 & I 5,118 & \\
\hline & 4571,040 & 83 & 55,072 & \\
\hline $\begin{array}{l}\text { A I interieur des classes de taux } \\
\text { butyreux } \ldots \ldots \ldots \ldots \ldots \ldots\end{array}$ & 7916,740 & 743 & 10,655 & $5,168 \times x$ \\
\hline Entre races $\ldots \ldots \ldots \ldots \ldots \ldots$ & I. 405,320 & $\begin{array}{r}143 \\
49\end{array}$ & 28,68 & \\
\hline A l'intérieur des races $\ldots \ldots \ldots \ldots$. & 6511,420 & $69+$ & $9,3^{8}$ & $3,05^{x x}$ \\
\hline 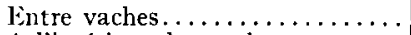 & 4367,100 & $43^{6}$ & 10,0 & \\
\hline A l'intérieur des vaches...$\ldots \ldots$ & 2144,300 & 258 & 8,31 & $\mathrm{I}, 20^{\mathrm{X}}$ \\
\hline
\end{tabular}

de taux butyreux présentent des différences significatives : il existe donc une liaison entre ces deux constituants, indépendamment du stade de lactation ;

$2^{\circ}$ Mais il y a, à 1'intérieur des classes de taux butyreux, une variation indépendante de la teneur en matières azotées ; elle résulte d'abord des variations entre vaches de races différentes, que nous montrait le graphique I, mais aussi, et surtout des différences entre vaches de la même race qui sont significativement supérieures aux variations intra-vaches: les teneurs en protéines des différentes vaches de la même race ne se répartissent donc pas de la même façon entre les diverses classes de taux butyreux.

Ces résultats confirment ceux queles auteurs scandinaves ont obtenus sur trois troupeaux différents (Bonnier et HANsson I946, HaNsson et Bonnier 1950, LARsson et al. r949), et nous permettent de séparer la variation de la teneur en matières azotées en deux parties : une variation liée à celle du taux butyreux (indépendamment du stade de lactation) et une variation indépendante, qui est de nature individuelle. Ce sont ces différences entre vaches qui masquent plus ou moins la liaison fondamentale entre teneur en protéines et taux butyreux, à 1'échelle de l'ensemble des laits individuels de la race et qu'il nous faut analyser maintenant.

\section{Inalyse des relations intra individuelles \\ Valeur et signification des coefficients de corrélation intra-individuels.}

I, tableau III donne, pour chaque vache, la valeur du coefficient de corrélation entre la teneur en protéines et le taux butyreux de la série d'échantillons prélevés au cours de sa lactation. Tous ces coefficients 
sont positifs, mais 8 d'entre eux ne sont pas significativement différents de zéro ; d'autre part la dispersion est beaucoup plus forte chez les Normandes ( 0,062 pour Joliette à 0,92 pour Pastille) que chez les Frisonnes $(0,3 \mathrm{I} 8$ à $0,8 \mathrm{OI})$. Cette distribution est sensiblement voisine de celles rapportées par les trois études antérieures de PRESSLER (I937) sur vaches pie noire, de Comberg (I95I) sur pie noires et de PIEL (I953) sur Brunes des Alpes dont le graphique 4 donne les histogrammes.

Cette liaison positive intra-individuelle entre taux butyreux et matières azotées provient du fait que ces deux constituants évoluent en moyenne dans le même sens au cours de la lactation, notamment pendant les deux premiers et les deux derniers mois oì sont concentrés presque la moitié de nos analyses (échantillons hebdomadaires). Par suite, la valeur du coefficient de corrélation individuel traduit le degré de similitude entre les deux courbes d'évolution, comme le montrent parfaitement les graphiques 3 correspondant aux valeurs extrêmes:

- la vache Pastille a présenté à partir du $2^{\mathrm{e}}$ mois de lactation une courbe d'enrichissement particulièrement régulière et accentuée, aussi bien pour le taux butyreux que pour la teneur en protéines, ce qui donne une répartition quasi linéaire des points sur le tableau de corrélation et un coefficient voisin de l'unité $(0,92)$;

- au contraire les deux courbes correspondantes de la vache Joliette sont très nettement différentes, voire même opposées dans certaines périodes, par exemple de la $4^{\mathrm{e}}$ à $1 \mathrm{a}$ Io ${ }^{\mathrm{e}}$ semaine et de $1 \mathrm{a} 22^{\mathrm{e}}$ à $1 \mathrm{a} 34^{\mathrm{e}}$; il en résulte un nuage de points circulaire et un coefficient de corrélation presque nul $(0,05)$.

Comme la teneur en matières azotées présente une évolution relativement régulière chez toutes les vaches, la valeur du coefficient de corrélation dépend en définitive des variations du taux butyreux. I'analyse des graphiques individuels nous montrerait ainsi que les huit coefficients non significatifs sont associés à des irrégularités du taux butyreux soit dans l'évolution générale au cours de la lactation (Joliette : 0,062, Astrée: 0, I25, Linotte : 0,330 , Rose : 0,340 ) soit d'un prélèvement à l'autre (Pietje : 0,318, Dalhia : 0,360, Tulipe : 0,379). Ces irrégularités peuvent être caractéristiques de 1'animal (grandes fluctuations journalières) ou déterminées par les facteurs climatiques alimentaires ou pathologiques ; enfin un petit nombre d'entre elles est probablement associé à une traite relativement incomplète, notamment chez certaines Normandes à temps de traite très long (ex. Joliette). Dans ces conditions nous ne pouvons pas dire si nos coefficients de corrélation phénotypiques intra-individuels rendent compte correctement de la liaison génétique (action des gènes communs) entre matières azotées et taux butyreux, et par suite en déduire qu'il existe entre vaches des différences dans l'intensité de cette liaison.

Pour permettre de telles conclusions il faut comparer des corrélations 


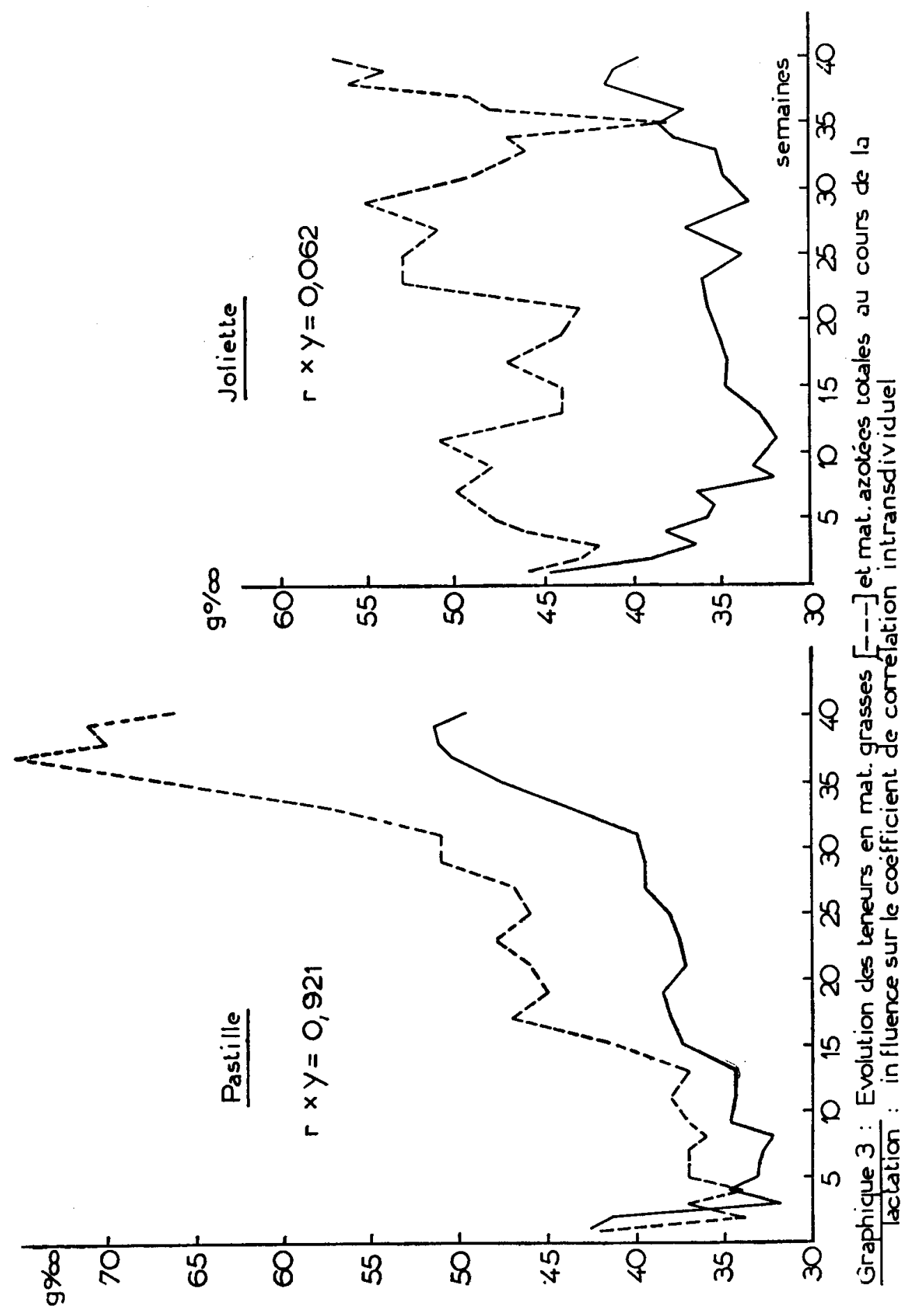




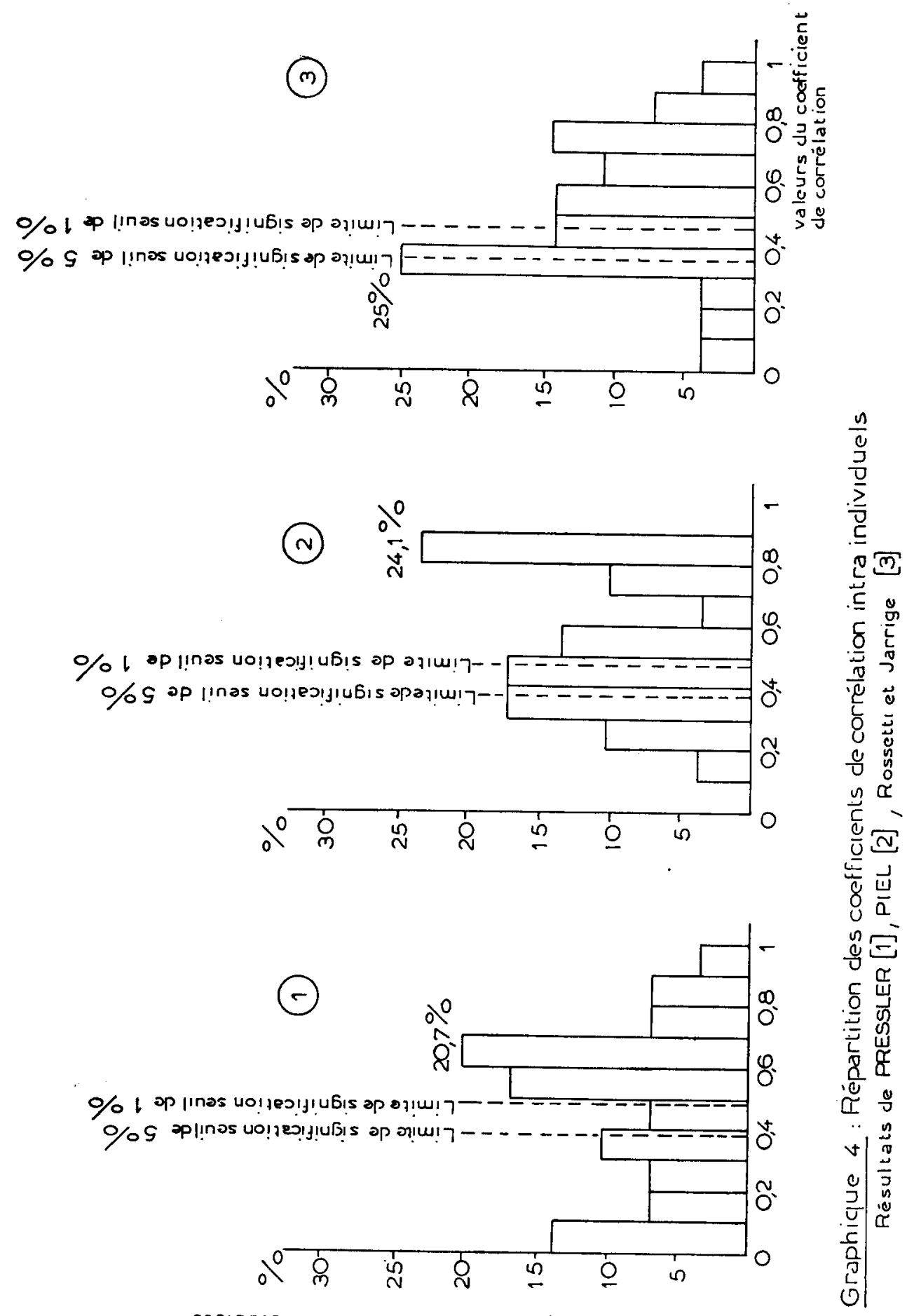


individuelles obtenues dans les mêmes conditions de milieu (climatalimentation) et débarrassées au maximum de l'influence des facteurs physiologiques qui sont prépondérants aux deux extrémités de la lactation; d'une part au cours de la phase d'appauvrissement des deux premiers mois, d'autre part à partir du $5^{\mathrm{e}}$ mois de gestation où le nouvel équilibre hormonal associé au développement du fœetus, détermine une augmentation de plus en plus nécessaire des deux teneurs en matières azotées et matières grasses : celles-ci présentent enfin dans ces deux périodes une étroite liaison négative avec la quantité de lait, alors qu'elles en sont pratiquement indépendantes dans l'intervalle (cf. JARRIGE et ROSSETTI I957 a).

C'est donc la corrélation calculée à partir des laits sécrétés entre le $3^{\mathrm{e}}$ mois après le vêlage et le $5^{\mathrm{e}}$ mois de gestation qui aura le plus de chances de rendre compte de la liaison génétique; pour avoir un grand nombre de valeurs, nous nous sommes adressés à un nouveau matériel et nous avons analysé les laits journaliers de 4 vaches.

\section{Différences individuelles dans l'intensité de la liaison}

Matériel. - Nous avons dosé les teneurs en matières grasses, matières azotées et lactose des laits journaliers de 4 vaches $\mathrm{du} 3^{\mathrm{e}}$ mois de lactation au tarissement. Le tableau ci-dessous donne la répartition des prélèvements et les graphiques 5, les moyennes arithmétiques hebdomadaires des teneurs.

\begin{tabular}{|c|c|c|c|c|c|c|c|c|}
\hline \multirow[b]{2}{*}{ Vache } & \multirow[b]{2}{*}{ Race. } & \multirow{2}{*}{$\begin{array}{c}\text { Lactation } \\
N^{\circ}\end{array}$} & \multirow{2}{*}{$\begin{array}{c}\text { Date } \\
\text { vêlage }\end{array}$} & \multirow{2}{*}{$\begin{array}{l}\text { Nombre } \\
\text { d'ana- } \\
\text { lyses }\end{array}$} & \multicolumn{2}{|c|}{$\begin{array}{l}\text { Début } \\
\text { des analyses } \\
\text { journaliéres }\end{array}$} & \multicolumn{2}{|c|}{$\begin{array}{c}\text { Fin } \\
\text { des analyses } \\
\text { journalières }\end{array}$} \\
\hline & & & & & Date & \begin{tabular}{|c} 
Nombre \\
de jours \\
après \\
vêlage
\end{tabular} & Date & $\begin{array}{l}\text { Nombre } \\
\text { de jours } \\
\text { après } \\
\text { saillie }\end{array}$ \\
\hline $\begin{array}{l}\text { Iglantine } \\
\text { Pervenche }\end{array}$ & $\begin{array}{l}\text { Frisonne } \\
\text { Frisonne }\end{array}$ & $\begin{array}{l}3 \\
3\end{array}$ & $\begin{array}{l}6 / 3 / 54 \\
5 / 3 / 54\end{array}$ & $\begin{array}{l}223 \\
187\end{array}$ & $\begin{array}{l}18 / 5 \\
18 / 5\end{array}$ & $\begin{array}{l}73 \\
74\end{array}$ & $\begin{array}{l}20 / \mathrm{I} / 55 \\
\mathrm{I} / \mathrm{I} / 55\end{array}$ & $\begin{array}{l}223 \\
187\end{array}$ \\
\hline $\begin{array}{c}\text { Actée } \\
\text { Joliette }\end{array}$ & $\begin{array}{l}\text { Normande } \\
\text { Normande }\end{array}$ & $\begin{array}{l}3 \\
3\end{array}$ & $\begin{array}{l}7 / 4 / 54 \\
7 / 4 / 54\end{array}$ & $\begin{array}{l}237 \\
237\end{array}$ & $\begin{array}{l}8 / 6 \\
8 / 6\end{array}$ & $\begin{array}{l}62 \\
5^{2}\end{array}$ & $\begin{array}{l}24 / 2 / 55 \\
24 / 2 / 55\end{array}$ & $\begin{array}{l}237 \\
237\end{array}$ \\
\hline
\end{tabular}

Ces 4 vaches dont nous avions étudié les lactations précédentes (cf. tableau III) ont été maintenues dans les mêmes conditions de milieu et d'alimentation ; malheureusement, en plus de la différence de 5-6 semaines dans les vêlages, nous avons eu trois autres facteurs de perturbation :

- en juillet, la teneur en matières azotées a diminué régulièrement jusqu'à des valeurs anormalement basses situées au-dessous du " minimum physiologique " (graph. 5) ; cette chute est vraisemblablement due à 
(II, I957) CONSTITUANTS AZOTÉS DES LAITS DE VACHE

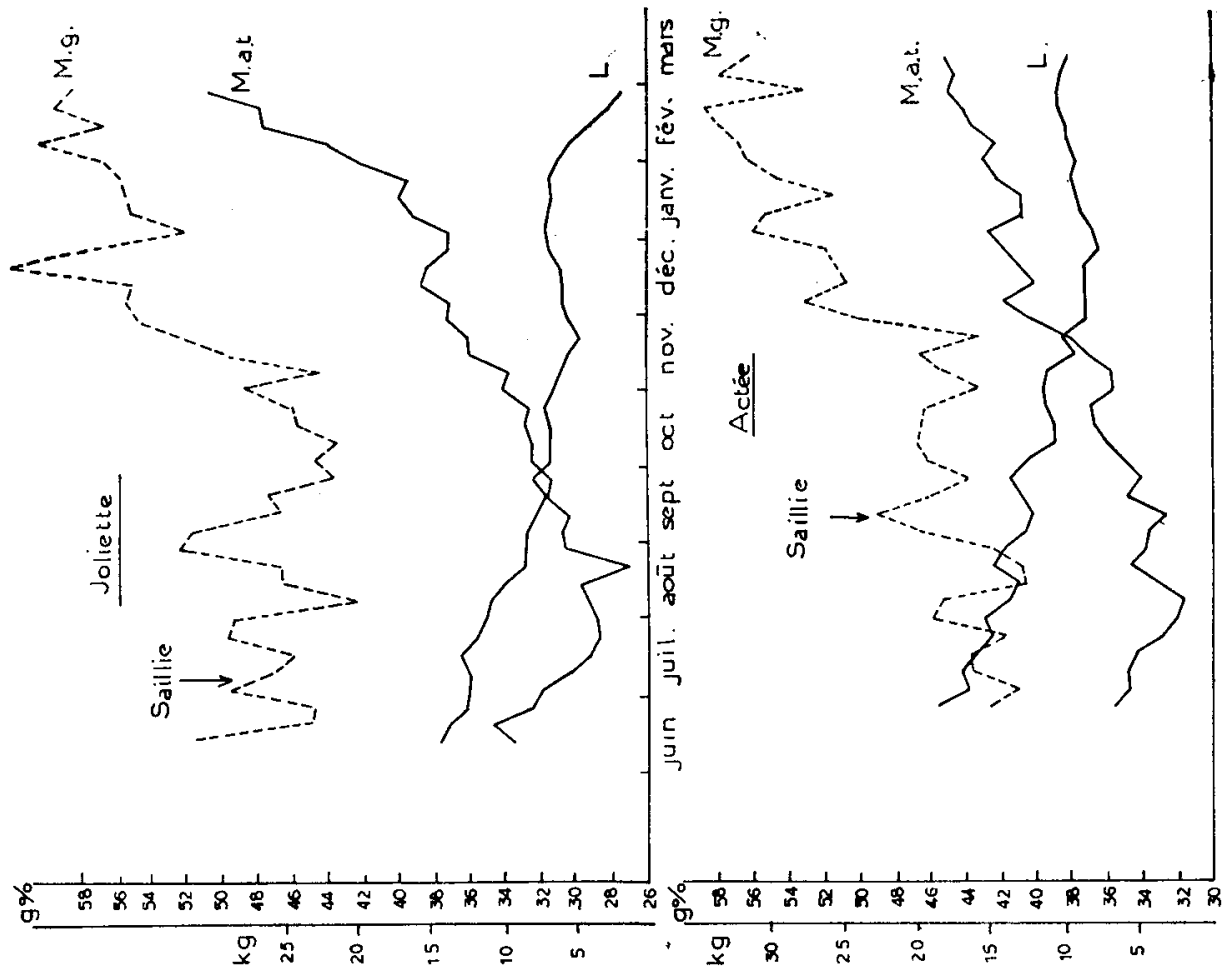

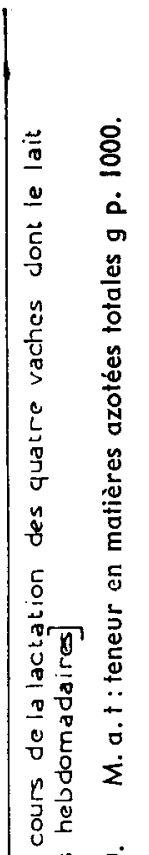

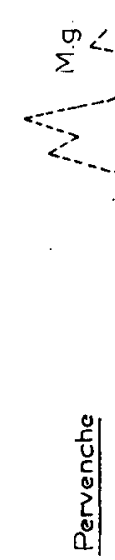

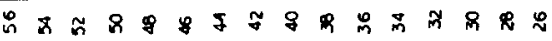
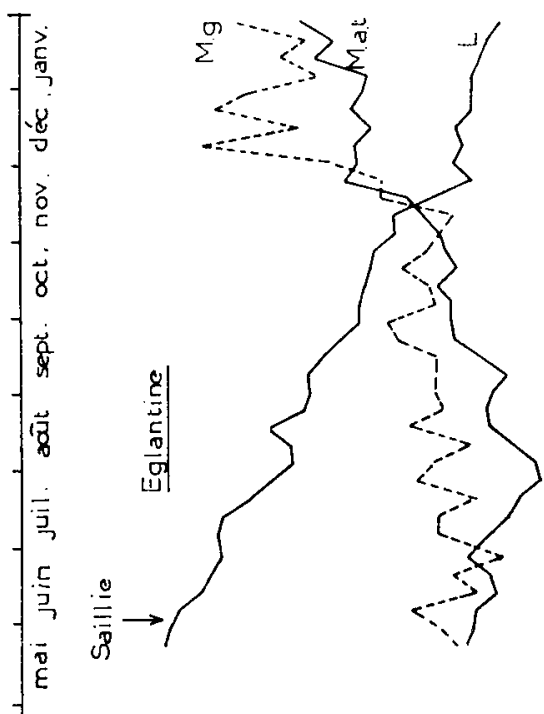
une sous-alimentation latente associée à la sécheresse (manque d'herbe, apport d'un fourrage vert de qualité médiocre) ;

- la vache Pervenche a subi une gastrotomie (corps étranger) le ro octobre, tandis que la vache Actée a été tarie par erreur un mois trop tôt;

- enfin, les analyses ont dû être interrompues pendant une semaine au mois d'août et les prélèvements effectués sur 48 heures du I I octobre au 28 novembre.

Si les coefficients de corrélation ont été vraisemblablement faussés en valeur absolue par ces différents incidents, ils demeurent néanmoins comparables d'un animal à l'autre, notamment dans la période moyenne de la lactation réalisée au pâturage de mai-juin à octobre.

Variabilité journalière. - Nous avons traduit les variations de la quantité et de la composition du lait d'un jour à 1'autre, par les différences journalières relatives :

$$
=\text { Ioo } \frac{\text { Valeur jour } \mathrm{J}+\mathrm{I} \text { - valeur jour } \mathrm{J}}{\text { Valeur jour } \mathrm{J}} \text {. }
$$

La moyenne de ces différences (tableau V) nous donne une estimation de la fluctuation journalière en dehors de l'évolution orientée au cours de la lactation.

TABLEAU V

Variabilité journalière de la quantité et de la composition du lait.

\begin{tabular}{|c|c|c|c|c|}
\hline & Vache & $\begin{array}{l}\text { Quantité } \\
\text { lait }\end{array}$ & $\begin{array}{c}\text { Teneur } \\
\text { matières } \\
\text { grasses }\end{array}$ & $\begin{array}{c}\text { Teneur } \\
\text { matières } \\
\text { azotées }\end{array}$ \\
\hline $\begin{array}{l}\text { Ensemble des } \\
\text { échantillons .... }\end{array}$ & $\begin{array}{c}\text { Eglantine } \\
\text { Pervenche } \\
\text { Actée } \\
\text { Joliette }\end{array}$ & $\begin{array}{c}\% \\
5,9 \\
6,2 \\
7,3 \\
7,9\end{array}$ & $\begin{array}{r}\% \\
8,8 \\
8,0 \\
10,6 \\
9,5\end{array}$ & $\begin{array}{l}\% \\
\\
2,1 \\
2,8 \\
2,6 \\
3,1\end{array}$ \\
\hline $\begin{array}{l}\text { Échantillons de } \\
\text { laits de pâturage }\end{array}$ & $\begin{array}{c}\text { Églantine } \\
\text { Pervenche } \\
\text { Actée } \\
\text { Joliette }\end{array}$ & $\begin{array}{l}5,3 \\
6,2 \\
7,4 \\
7,8\end{array}$ & $\begin{array}{r}8,5 \\
8, \mathrm{I} \\
\mathrm{IO}, 4 \\
\mathrm{I} \mathrm{I}, 4\end{array}$ & $\begin{array}{l}2,0 \\
2,8 \\
2,3 \\
3,3\end{array}$ \\
\hline
\end{tabular}

Que ce soit pour l'ensemble des laits de chaque vache ou pour ceux produits dans la phase moyenne (juin à octobre), nous arrivons à deux conclusions importantes :

- les fluctuations de la teneur en matières azotées sont très limitées $(2,5$ p. Ioo en moyenne, du même ordre que le lactose $), 2$ à 3 fois plus faibles que celles de la quantité de lait (6 à 7 p. Ioo) et 3 à 4 fois plus 
que celles du taux butyreux ( 8 à Io p. Ioo) qui est le constituant organique de loin le plus variable;

- il existe des différences très nettes entre nos quatre vaches : par exemple : Joliette est beaucoup plus irrégulière qu'Eglantine aussi bien pour la teneur en matières azotées que pour la quantité de lait et le taux butyreux.

\section{Corrélations entre taux butyreux $(y)$ et teneur en matières azotées $(x)$.}

Nous avons calculé (tableau VI) des coefficients de corrélation pour l'ensemble des laits et, pour différentes tranches de lactation en prenant, cette fois-ci, comme variable indépendante, la teneur en matières azotées totales qui est beaucoup plus régulière et traduit en partie le stade de lactation.

TABLEAU VI

Coefficients de corrélation entre la teneur en matières azotées $(x)$ et le taux butyreux $(y)$ des laits individuels journaliers.

\begin{tabular}{|c|c|c|c|c|c|c|}
\hline & & & \multicolumn{4}{|c|}{ Nom de la vache } \\
\hline & & & Errlantine & Pervenche & Actée & Joliette \\
\hline & 0,06 & 0,15 & $-0,22^{\times}$ & $0, \mathrm{I} 5$ \\
\hline \multicolumn{3}{|c|}{$\begin{array}{l}\text { Avant la saillie } \ldots \ldots \ldots \ldots \ldots \\
\text { I }{ }^{\text {er }} \text { et } 2^{\text {e }} \text { mois de gestation } \ldots \ldots\end{array}$} & $-0,27^{x}$ & $-0,15$ & 0,14 & $-0,01$ \\
\hline $3^{\mathrm{e}}$ et $4^{\mathrm{e}}$ & n & $" \quad \ldots \ldots$ & 0,15 & $0,30^{x}$ & $0,21^{x}$ & 0,17 \\
\hline $5^{\mathrm{e}}$ et $6^{\mathrm{e}}$ & $"$, & - & $0,64^{\times x}$ & $0,5^{2 \times x}$ & $-0,07$ & $0,29^{x}$ \\
\hline & & $\ldots$ & 0,00 & $0,48^{* x}$ & & $0,29^{\circ}$ \\
\hline linsemble & des lai & $\ldots \ldots \ldots \ldots$ & $0,67^{x \ldots}$ & $0,8 \mathrm{I}^{\times *}$ & $0,58^{\times x}$ & $c, 59^{\times \times}$ \\
\hline linsemble & des lai & e pâturagge.. & $0,257^{x}$ & $0,493^{x}$ & $0, \mathrm{I} 55$ & - o, Ior \\
\hline
\end{tabular}

Les valeurs obtenues pour l'ensemble des laits sont forterrent positives parce qu'elles résultent, une fois de plus, de l'augmentation simultanée des deux constituants en fin de lactation : la liaison faible ou même négative avant la saillie et au cours des deux premiers mois de gestation, devient positive au cours des $3^{\mathrm{e}}$ et $4^{\mathrm{e}}$ mois et très étroite au $5^{\mathrm{e}}$ et $6^{\mathrm{e}}$. Les valeurs individuelles des deux premières tranches de gestation, sont d'ailleurs relativement artificielles et dépendent par trop de l'échantillonnage, notamment de la place de la saillie par rapport à la dépression estivale de la teneur en protéines (graph. 5).

Par contre, nous éliminons ces erreurs de découpage, en considérant 1'ensemble des laits de pâturage (de mai-juin à fin octobre) qui correspondent en gros à l'ensemble des trois premières tranches et sont donc produits dans des conditions physiologiques très comparables et des conditions de milieu identiques. Nous obtenons des coefficients de corrélation significativement différents (dernière ligne du tableau VI) d'autant 
plus élevés que la variabilité journalière du taux butyreux (tableau V) est plus faible : Pervenche 8 , I p. Ioo et $0,493^{\times x}$, Eglantine 8,4 p. Ioo et $0,257^{\times}$, Actée Io,4 p. Ioo et o,I55, Joliette II,4 p. Ioo et o,IoI.

Pour préciser la nature de la liaison dans cette période nous avons analysé la variance du taux butyreux en relation avec la teneur en matières azotées (tableau VII) ; nous avons pris comme intervalle de classe de celles-ci la moitié de son écart type, ce qui nous a donné sensiblement le même nombre de classes pour les 4 vaches ( 8 pour Pervenche, 9 pour Actée et Joliette, II pour Eglantine) ; nous avons d'autre part calculé le rapport de corrélation qui exprime la liaison de la façon la plus générale sans impliquer d'hypothèse sur sa nature; c'est par définition le rapport :

somme des carrés du taux butyreux entre classes de matières azotées Somme des carrés

\section{TABleau VII}

Analyse de la variance intra individuelle du taux butyreux en relation avec la teneur en matières azotées totales.

\begin{tabular}{|c|c|c|c|c|c|c|c|c|}
\hline Nom de la vache....$\ldots \ldots$ & \multirow{2}{*}{\multicolumn{2}{|c|}{$\begin{array}{l}\text { Églantine } \\
\qquad \begin{array}{l}0,257^{\times} \\
0,308\end{array}\end{array}$}} & \multirow{2}{*}{\multicolumn{2}{|c|}{$\begin{array}{l}\text { Pervenche } \\
0,493^{\mathrm{xx}} \\
0,635\end{array}$}} & \multirow{2}{*}{\multicolumn{2}{|c|}{$\begin{array}{l}\text { Actée } \\
0,155 \\
0,264\end{array}$}} & \multirow{2}{*}{\multicolumn{2}{|c|}{$\begin{array}{r}\text { Joliette } \\
-0,1 \text { I I } \\
0,2 \text { I } 5\end{array}$}} \\
\hline $\begin{array}{l}\text { Coefficient de corrélation..... } \\
\text { Rapport de corrélation ...... }\end{array}$ & & & & & & & & \\
\hline Origine de la variation ....... & $\begin{array}{l}\text { Nb. } \\
\text { degrés } \\
\text { liberté }\end{array}$ & $\begin{array}{l}\mathrm{Va-} \\
\text { riance }\end{array}$ & $\begin{array}{l}\text { Nb. } \\
\text { degrés } \\
\text { liberté }\end{array}$ & $\begin{array}{l}\mathrm{Va}- \\
\text { riance }\end{array}$ & $\begin{array}{l}\text { Nb. } \\
\text { degrés } \\
\text { liberté }\end{array}$ & $\begin{array}{l}\mathrm{Va-} \\
\text { riance }\end{array}$ & $\begin{array}{l}\text { Nb. } \\
\text { degrés } \\
\text { liberté }\end{array}$ & $\begin{array}{l}\mathrm{Va}- \\
\text { riance }\end{array}$ \\
\hline $\begin{array}{l}\text { Régression linéaire } \ldots . . . \ldots \\
\text { Déviations par rapport à la } \\
\text { ligne droite } \ldots \ldots \ldots \ldots \ldots\end{array}$ & 9 & $4, \mathrm{I} 3$ & $\begin{array}{l}\mathbf{I} \\
6\end{array}$ & 85,97 & $\begin{array}{l}\mathrm{I} \\
7\end{array}$ & 16,84 & $\begin{array}{l}\text { I } \\
7\end{array}$ & 19,97 \\
\hline Totales entre classes...... & Io & $\mathrm{r} 2, \mathrm{I} 8$ & 7 & 86,05 & 8 & 22,49 & 8 & 22,42 \\
\hline Résiduelle (erreur) $\ldots \ldots \ldots$ & $\mathrm{I}_{42}$ & 8,17 & I $3^{8}$ & I 3,97 & I $2 \mathrm{I}$ & $\mathrm{r} 9,88$ & 122 & 30,36 \\
\hline Total général $\ldots \ldots \ldots \ldots$ & I 52 & & 145 & & I 29 & & I 30 & \\
\hline
\end{tabular}

Ie tableau nous montre que, seule, Pervenche présente une liaison significative mais non linéaire; chez Eglantine la liaison approche le seuil de signification et serait peut-être linéaire; enfin il n'y a pas de liaison chez les deux autres vaches.

En définitive, il existerait donc des différences individuelles : (schématisées par le graph. 6) :

- d'abord dans l'amplitude de la variation journalière du taux butyreux (ainsi que des autres constituants) ;

- ensuite dans la part de cette variation totale qui est liée à celle 
de la teneur en matières azotées : en admettant que le rapport de corrélation en soit une estimation valable, elle serait de $2 \mathrm{r}, 5 \mathrm{p}$. roo pour Joliette, 26,4 p. roo pour Actée, 30,8 p. Ioo pour Eglantine et $63,5 \mathrm{p}$. Ioo pour Pervenche ;

- dans l'intensité de la liaison entre les deux teneurs et, par suite, à titre d'hypothèse, dans le nombre de gènes communs aux deux sécrétions.

Ces conclusions n'ont naturellement de valeur que dans la mesure

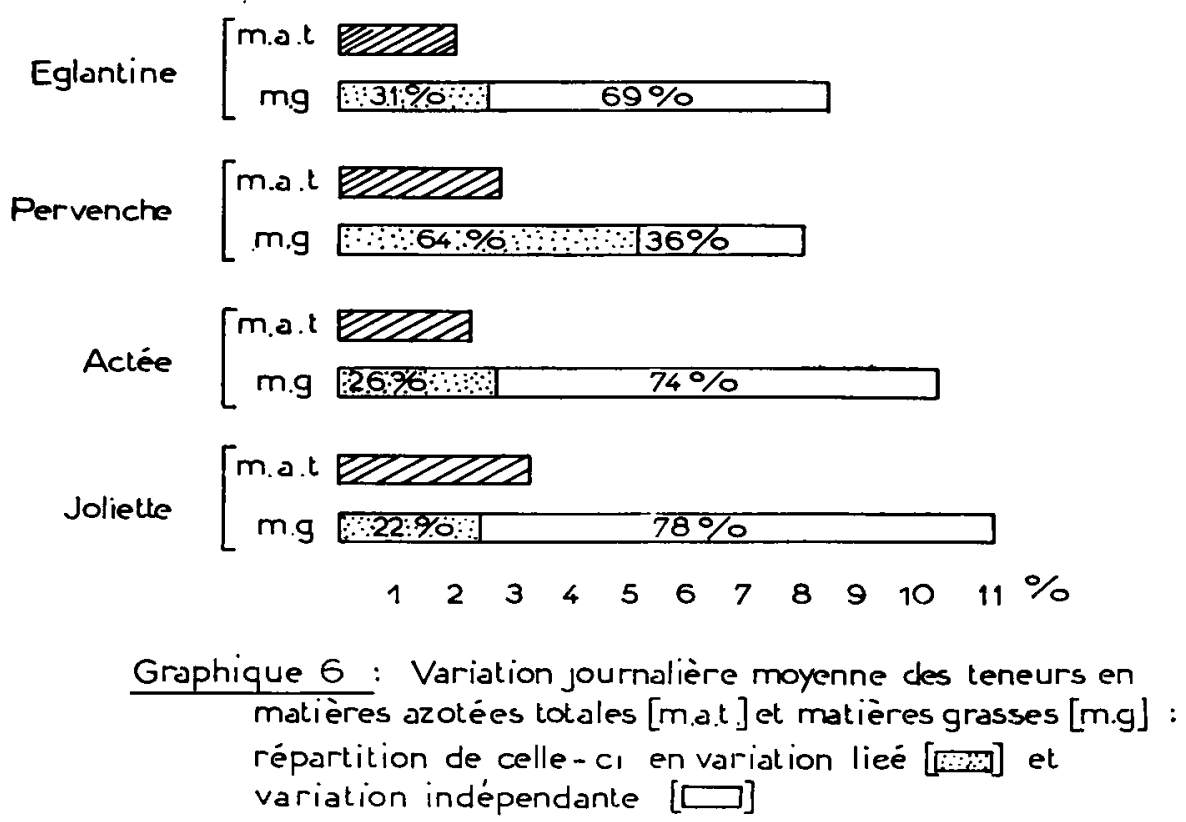

où nous admettons que les corrélations phénotypiques que nous avons calculées traduisent les liaisons génétiques fondamentales avec la même approximation chez nos quatre animaux. Nous comptons les vérifier ultérieurement en appliquant à des animaux maintenus dans des conditions de milieu moins variables, ces méthodes d'interprétation qui, elles, demeurent valables.

\section{Relation: interindividuelles}

Existence d'une liaison.

Le graphique 7 où nous avons représenté chaque vache par ses valeurs moyennes pondérées en matières grasses et matières azotées totales, nous montre immédiatement que, en moyenne, la teneur en matières azotées 
augmente avẹc le taux butyreux. En supposant que cette liaison interindividuelle est linéaire, nous obtenons les coefficients suivants :

\begin{tabular}{|c|c|c|c|c|}
\hline & \multicolumn{2}{|c|}{$\begin{array}{c}\text { Matières azotées totales }(y) \\
\text { Matières grasses }(x)\end{array}$} & \multicolumn{2}{|c|}{$\begin{array}{l}\text { Caséine }\left(y^{\prime}\right) \\
\text { Matières grasses }(x)\end{array}$} \\
\hline . & Corrélation & Régression & Corrélation & Régression \\
\hline $\begin{array}{l}\text { Vaches frisonnes (I6).. } \\
\text { Vaches normandes (II). } \\
\text { Ensemble des } 27 \text { vaches. }\end{array}$ & $\begin{array}{l}0,783^{\times \times x} \\
0,37^{2} \\
0,867^{\times x}\end{array}$ & $\begin{array}{l}y=0,495 x+14,90 \\
y=0,242 x+25,90 \\
y=0,476 x+15,63\end{array}$ & $\begin{array}{l}0,816^{\times x} \\
0,213 \\
0,85^{x \times}\end{array}$ & $\begin{array}{l}y=0,478 x+8,27 \\
y=0,213 x+19,06 \\
y=0,394 x+\text { I I,I } 7\end{array}$ \\
\hline
\end{tabular}

Ia corrélation est hautement significative pour les Frisonnes mais non pour les normandes en raison de leur dispersion; elle le redevient pour l'ensemble des vaches parce que la teneur en matières azotées augmente en même temps que le taux butyreux quand on passe des Frisonnes (t. b. : 34,o, m. a. t. : 3I, 4) aux Normandes (t. b. : 43,4, m. a. t. : $36,4)$. En moyenne quand le taux butyreux augmente d'un point entre deux individus, la teneur en matières azotées augmente mais d'une quantité beaucoup plus faible : 0,49 chez les Frisonnes et 0,24 chez les Normandes; il en résulte une diminution du rapport $\frac{\text { matières azotées }}{\text { taux butyreux }}$.

Nous avons admis en accord avec KRIZENECKY (I943) que la liaison entre les deux teneurs, est linéaire et de la forme $y=a x+b$ et par suite que leur rapport est une fonction hyperbolique du taux butyreux $\frac{y}{z}=a+\frac{b}{x}$. Nous constatons que ces courbes rendent assez bien compte des deux distributions (graph. 7) mais nous n'avons pas assez de valeurs pour démontrer que la régression linéaire est la meilleure représentation de la liaison : c'est là d'ailleurs un problème relativement formel si on considère l'importance des écarts individuels.

\section{Différences individuelles.}

Pour un même taux butyreux nous avons des différences allant jusqu'à $5 \mathrm{~g}$ dans la teneur en matières azotées totales, par exemple de 34,6 (Joliette) à 39,8 (Actée) pour $47 \mathrm{~g}$ de matières grasses ; réciproquement à la même teneur en matières azotées, de $35 \mathrm{~g}$ par exemple correspondent des taux butyreux allant de 38,5 (Cabale) à 47,2 (Joliette). Ira teneur en protéines n'augmente donc pas obligatoirement avec le taux butyreux quand on passe d'une vache à l'autre à l'intérieur de la même race.

Ces différences se traduisent par de larges variations du rapport des moyennes pondérées $\frac{m . a . t .}{t . b .}$ et $\frac{\text { caséine }}{t . b \text {. }}$ que nous pouvons résumer de la façon suivante : 
(II, I957) CONSTITUANTS AZO'TÉS DES LAITS DE VACHE
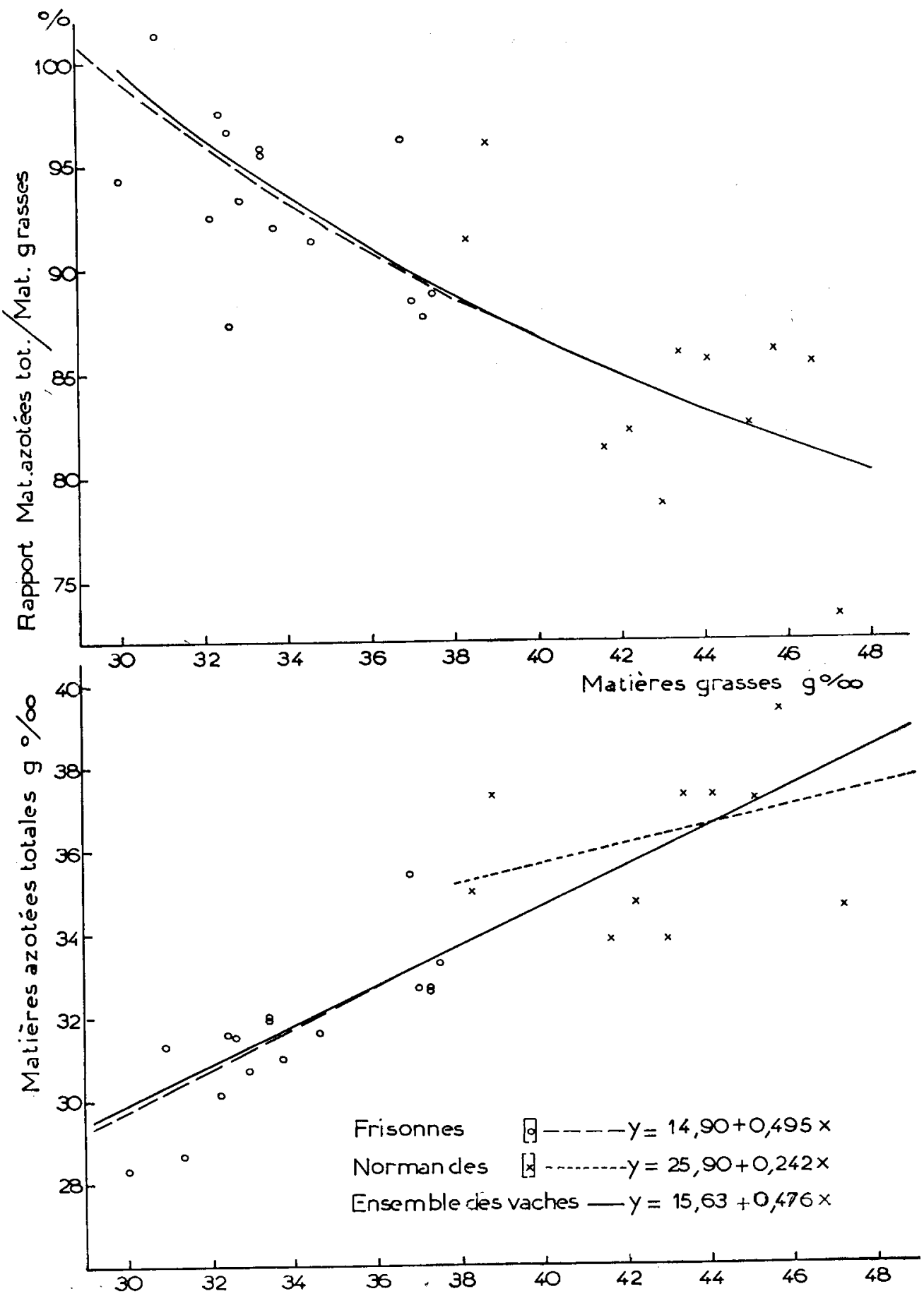

Graph 7 : Relations interindividuelles entre teneurs en matières azotées totales et taux butyreux. 


\begin{tabular}{|c|c|c|c|c|c|c|}
\hline & \multicolumn{3}{|c|}{$\frac{\text { Matières azotées totales (pondérées) }}{\text { taux butyreux (pondéré) }}$} & \multicolumn{3}{|c|}{$\frac{\text { Caséine (pondérée) }}{\text { taux butyreux (pondéré) }}$} \\
\hline & Limites & Moyenne & $\begin{array}{l}\text { Ecart } \\
\text { type }\end{array}$ & Limites & Moyenne & $\begin{array}{l}\text { Ecart } \\
\text { type }\end{array}$ \\
\hline $\begin{array}{l}\text { Vaches frisonnes... } \\
\text { Vaches normandes. }\end{array}$ & $\begin{array}{l}87,7 \text { à ror, } 3 \\
73,3 \text { à } 96, \mathbf{1}\end{array}$ & $\begin{array}{l}93,48 \\
84,30\end{array}$ & $\begin{array}{l}3,84 \\
6,06\end{array}$ & $\begin{array}{l}69,3 \text { à } 78,3 \\
57,5 \text { à } 75,0\end{array}$ & $\begin{array}{l}74,4 \mathrm{I} \\
65,55\end{array}$ & $\begin{array}{l}3,15 \\
4,94\end{array}$ \\
\hline
\end{tabular}

Nous constatons que les Normandes présentent une dispersion beaucoup plus élevée que les Frisonnes et une moyenne nettement plus faible : d'une race à l'autre la teneur en protéines augmente moins vite

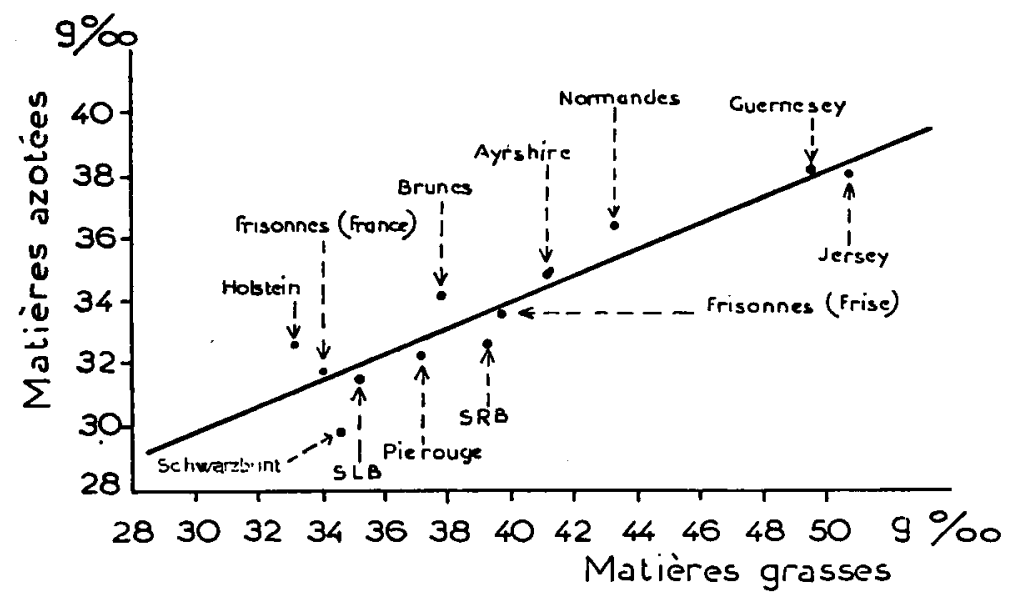

Graphique 8 Ebauche d'une corrélation interraces entre teneur en matières azotées et taux butyreux (d'après nos résultats et ceux du tableau 1)

que le taux butyreux ce qui est en accord avec les observations de GaInes et Overman (I938) aux U. S. A., et de Larsson et al. (r949) en Suède (cf. graph. 8).

Dans le tableau VIII nous avons fait une analyse hiérarchique de la variance des 323 valeurs individuelles du rapport observées entre la fin du $2^{\mathrm{e}}$ mois et la fin du $8 \mathrm{e}$; nous avons en effet montré précédemment (JARRIGE et ROSSETTI I957a) que le rapport demeurait sensiblement constant dans cette période, alors qu'il diminuait de façon significative dans les deux premiers mois et fluctuait fortement dans les deux derniers. Nous avons successivement classé les valeurs par stades de lactation (3 périodes de 2 mois), par races et par vaches.

Cette analyse nous confirme d'abord que le rapport m. a. t./t. b. n'évolue pas au cours de la période de lactation envisagée et varie de 


\section{TABLEAU VIII}

Analyse hiérarchique de la variance du rapport:

$\frac{\text { Teneur en matières azotées totales } X}{\text { Taux butyreux }}$

\begin{tabular}{|c|c|c|c|c|}
\hline Cause de variation & $\begin{array}{l}\text { Somme } \\
\text { des carrés }\end{array}$ & $\begin{array}{l}\text { Degrés } \\
\text { de liberté }\end{array}$ & Variance & Rapport \\
\hline Total ............... & 40370,205 & 323 & & \multirow[b]{3}{*}{0,594} \\
\hline Entre stades de lactation........ & 148,925 & 2 & 74,465 & \\
\hline $\begin{array}{l}\text { A l'intérieur des stades de lactation } \\
\text { Entre races (à l'intérieur des stades }\end{array}$ & 40221,280 & 321 & I 25,299 & \\
\hline de lactation).................... & 8826,510 & 3 & 2942, I 70 & \multirow{2}{*}{$29,803^{\times x}$} \\
\hline 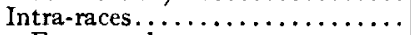 & 31394,770 & 318 & 98,720 & \\
\hline 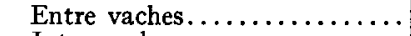 & 16097,220 & 75 & 214,629 & \multirow{2}{*}{$3,4 \circ 9^{x x}$} \\
\hline Intra-vaches $\ldots \ldots \ldots \ldots \ldots \ldots$ & 15297,550 & 243 & $62,95^{2}$ & \\
\hline
\end{tabular}

façon hautement significative d'une race à l'autre; elle nous démontre ensuite que les variations inter-vaches sont supérieures aux variations intra-vaches et que ce rapport est donc une caractéristique individuelle.

\section{DISCUSSION}

\section{Existence d'une liaison positive à trois niveaux}

Au cours de l'exposé détaillé de nos résultats nous avons montré qu'il existait entre la teneur en protéines et le taux butyreux une liaison positive à trois niveaux.

- La première apparaît au niveau intraindividuel pour l'ensemble des laits prélevés à intervalles réguliers au cours de lactation d'une même vache; elle traduit le fait que les deux constituants ont en moyenne une évolution semblable et sa valeur est avant tout fonction de l'échantil onnage, et de la régularité du taux butyreux. Calculée à partir des laitsjournaliers de la phase moyenne de la lactation, elle nous a cependant permis de montrer que l'intensité de la liaison intraindividuelle pourrait varier d'un individu à l'autre.

- La liaison interindividuelle dans laquelle chaque vache est représentée par ses valeurs moyennes pondérées (graph. 7) est de loin la plus importante. Entre individus de la même race, la teneur en protéines augmente en moyenne avec le taux butyreux mais à un rythme beaucoup plus faible; les coefficients de régression de 0,495 pour les Frisonnes et 0,242 pour les Normandes n'ont qu'une valeur très contingente en raison de notre échantillonnage limité, mais sont du même ordre que ceux rapportés pour d'autres races (tableau II), notamment par 
AUriol (I956) pour les 235 premières vaches Pie Rouge de 1'Est de son enquête sur les aptitudes fromagères $(0,282)$.

- Enfin les différences entre les Normandes et les I'risonnes seraient un élément d'une liaison inter-races qui ressort du graphique 8, nous y avons représenté chaque race par la moyenne des valeurs individuelles obtenues par les auteurs du tableau I, notamment GaInes et Overman (I938). Bien que chacune de ces populations (à 1'exception des Pie Rouge) soit trop limitée pour être vraiment représentative de la race, elles se répartissent très étroitement autour d'une ligne de régression :

$$
y=0,4 \operatorname{I} 2 x+\operatorname{I} 7,49 \quad r_{x y}=0,947 .
$$

Les corrélations positives obtenues sur des ensembles de laits individuels et, a fortiori, des laits de mélange, ne sont que la résultante plus ou moins composite de ces trois liaisons fondamentales. Leur valeur (tableau I) dépend avant tout du nombre et de la répartition des échantillons entre individus, race et stades de lactation (cf. graph. I). Elles conservent un intérêt certain pour mettre en évidence les modifications de la composition et des qualités technologiques des laits de troupeaux ou de laiterie en fonction de 1'année, la saison, l'alimentation et l'orientation de la sélection. C'est ainsi que VALEN (I953) étudiant les relations entre teneur en protéines $(y)$ et matières grasses $(x)$ des laits de 53 laiteries disséminées dans toute la Norvège, constate d'une part des variations très importantes d'une région à l'autre (race...) mais, d'autre part, une différence systématique entre les laits produits au pâturage et ceux produits à l'étable; la liaison est beaucoup plus forte chez ces derniers comme le montrent les moyennes nationales suivantes :

$$
\begin{array}{lll}
\text { laits de pâturage } & y=2,4 \mathrm{I}+0,24 x & \gamma_{x y}=0,48 \pm 0,09 \\
\text { laits d'étable } & y=\mathrm{I}, 63+0,47, x & r_{x y}=0,77 \pm 0,09 .
\end{array}
$$

Par contre ce type de calcul ne permet pas d'interpréter les relations entre les deux constituants et, surtout, d'en tirer des conclusions génétiques comme l'ont fait trop d'auteurs qui auraient dû méditer sur les dangers et les limites de la méthode des corrélations qu'on ne saurait mieux résumer que 1'HERITIER (I949) : " L'existence d'une corrélation plus ou moins intense entre deux grandeurs est un fait mathématique dont l'interprétation sur le plan causal peut être extrêmement variable et doit être discutée et, si possible analysée dans chaque cas particulier. "(In Les méthodes statistiques, p. 66).

\section{Amélioration de la teneur en proteines par la sélection sur le taux butyreux}

De la liaison interindividuelle systématiquement positive nous pouvons conclure que dans l'ensemble les vaches à taux butyreux élevé, ont 
également un lait plus riche en protéines que la moyenne et surtout, que les vaches à faible taux butyreux. Eñ éliminant ces dernières pour sélectionner les premières nous devrions augmenter la teneur en protéines en même temps que le taux butyreux puisqu'elle a une héritabilité aussi élevée sinon plus (HANCOCK, I953). Pour vérifier cette hypothèse, il faut comparer des générations successives d'animaux, notamment des couples mère-fille, comme le fait actuellement Aurior, (r956). N'oublions pas en effet, que les corrélations interindividuelles calculées par les différents auteurs (tableau II) ne permettent pas de conclusions génétiques absolues puisque ce sont des corrélations phénotypiques portant, de plus, sur un nombre de vaches en général très réduit : on peut cependant penser que l'interférence des facteurs externes a été relativement faible, car les animaux étaient en général maintenus dans des conditions de milieu comparables (année, région...).

A défaut de preuves directes tout au moins pour l'instant, nous pouvons voir un argument indirect dans la comparaison de notre échantillon de I6 Frisonnes à celui étudié en Frise par JANSE et al. (I954); ce dernier présente en moyenne $5.7 \mathrm{~g}$ de matières grasses de plus $(39,7$ p. I ooo contre 34,0 p. I ooo) et $\mathrm{r}, 9 \mathrm{~g}$ de matières azotées $(33,6 \mathrm{p}$. I ooo contre $3 \mathrm{r}, 7 \mathrm{p}$. I 0oo). Il semble donc que l'amélioration du taux butyreux poursuivie en Frise s'accompagnerait d'une augmentation de la teneur en protéines qui serait en gros trois fois plus faible. Tout en n'attribuant pas une grande signification à ce rapport d'un tiers, il faut quand même souligner qu'il est tout à fait comparable à la valeur moyenne des coefficients de régression interindividuels, dont il justifierait l'intérêt génétique.

Une telle amélioration serait d'ailleurs déjà très satisfaisante si on considère que les teneurs individuelles en matières azotées ont une dispersion relativement étroite (cf. JARRIGE et RossETTI, I957 $b$ ) et ne présentent pas une liaison stricte avec le taux butyreux. Nous constatons ainsi (tableau III) que la différence entre la teneur réelle et celle calculée à partir de l'équation de régression est en moyenne de $r, 8 \mathrm{~g}$ pour nos Normandes et de 0,7 pour nos Frisonnes; cette dernière valeur est faible mais il n'en reste pas moins qu'on en ignore toujours le signe. Il apparaît donc difficile d'estimer, avec un degré d'approximation assuré, la teneur en matières azotées à partir du taux butyreux pondéré (même avec des lois de régression calculées sur des populations importantes) d'autant plus que celui-ci est lui-même entaché dans la pratique d'une erreur qui peut être importante ne serait-ce qu'en raison de ses fluctuations journalières. En définitive, pour augmenter rapidement la teneur en protéines des laits, il faut faire une sélection directe en contrôlant les différences individuelles par des dosages effectués au laboratoire (cf. JARRIGE et ROSSETTI I957 b). 
Ce contrôle nécessairement onéreux ne peut être envisagé que dans les conditions où il sera remboursé par une plus value du lait ou des reproducteurs, c'est-à-dire seulement dans les régions strictement fromagères, car l'amélioration de la teneur en caséine s'y matérialise par une augmentation immédiate du poids de fromage fabriqué, à la ferme ou à la coopérative. Par contre, une telle amélioration ne serait pas actuellement rémunérée dans les zones productrices de laits de consommation, bien qu'elle soit très souhaitable du point de vue nutritionnel; il faut .donc s'y contenter de l'augmentation indirecte, lente mais gratuite, liée à celle du taux butyreux. Il en est de même dans toutes les régions de France où on "fait " du fromage en même temps que du beurre et du lait de consommation, d'autant plus que les matières grasses y sont en général beaucoup mieux payées que les matières azotées. Einfin, celles-ci n'intéressent pas les beurreries (Normandie, Charente) et présentent une teneur suffisante dans le lait écrémé destiné aux jeunes animaux, surtout si on la rapporte à la valeur énergétique; il est par contre inutile de chercher à les diminuer dans ces régions, comme le propose LoNkA (r947) pour la Finlande, car les méthodes modernes d'exploitation fourragère (prairies temporaires, pâturage rationné, ensilage) permettent d'apporter aux vaches, en toute saison, une ration riche en protéines. Dans les zones où il est difficile d'utiliser ces méthodes on pourrait théoriquement envisager une sélection divergente, visant à améliorer le taux butyreux, sans augmenter la teneur en matières azotées, voire même en la diminuant; il existe en effet des animaux qui répondent à ce but, et dont certains pourraient avoir en outre, une liaison protéines-matières grasses plus lâche.

Quelles que soient les possibilités économiques actuelles, il n'en reste pas moins que les protéines sont les constituants du lait les plus intéressants du point de vue nutritionnel: il est donc souhaitable d'augmenter leur teneur ou, tout au moins, de prévenir la diminution de celleci. En Grande-Bretagne on constate, en effet, que l'extrait sec dégraissé (et, par là même, la teneur en matières azotées) des laits a tendance à diminuer depuis 25 ans et que la fréquence des teneurs inférieures au minimum légal $\left(85^{\circ} / 00\right)$ augmente à la fin de $1^{\prime h}$ hiver et à la mi-été (cf. Provan I955 - KAY I955). Ces laits "illégaux ", également signalés en France aux mêmes époques (EYyrard, Hurel et BoIsSEAU I946) sont en général produits pendant les périodes de sous-alimentation énergétique (RowLAND I946) par les vaches secrétant un lait toujours relativement pauvre. Le contrôle de la teneur en matières grasses permet heureusement de déceler et d'éliminer la plupart de ces animaux; on peut donc dire, à la limite, que le principal intérêt nutritionnel de la sélection sur le taux butyreux est d'améliorer la teneur en protéines. 


\section{Composition du lait et valeur fromagère}

Nous ne pouvons actuellement envisager de sélection directe sur la teneur en protéines que dans les régions strictement fromagères (Doubs, Jura, Savoie, Haute-Savoie, Cantal...).

Il nous faut donc discuter plus précisément les relations entre la composition du lait et sa valeur fromagère, et ceci sous trois aspects : rendement, qualité, coût énergétique.

Rendement en fromage. - Comment exprimer le rendement fromager d'un lait à partir de sa richesse en matières grasses et matières azotées ? Telle est la question que nous avons posée à M. G. MocQUot, Directeur de la Station Centrale de Microbiologie et de Recherches Laitières, qui nous en a montré la complexité, en raison du grand nombre des facteurs qui déterminent le rendement du lait en fromage frais ; il en distingue trois catégories :

- le type de fromage fabriqué, la teneur en eau étant très variable, selon qu'on fabrique par exemple des pâtes molles ou des pâtes dures ;

- la teneur en caséine et graisse du lait, puisque ces deux constituants représentent plus de $90 \mathrm{p}$. Ioo de l'extrait sec du fromage tout au moins dans les fromages à pâte dure ; la caséine joue un rôle prépondérant car elle retient dans le fromage de 5 à 7 fois plus d'eau que les matières grasses : en moyenne pour I $\mathrm{kg}$ de caséine dans le lait on obtient I,5 kg d'eau dans le Cheddar (cf. Van SLYKE et PRICE I952) ;

- les propriétés du coagulum obtenu par action de la présure (et des bactéries lactiques) sur le lait: un coagulum mou laisse passer, toutes choses égales, plus de graisse et plus de caséine dans le sérum qu'un coagulum ferme (influence de la teneur en calcium, de la pasteurisation, la " nature de la caséine $")$.

Des laits ayant les mêmes teneurs en graisse et caséine donnent ainsi des poids de fromage frais très variables, non seulement avecle type de fromage, mais encore suivant le procédé de fabrication, différent d'une fromagerie et d'une année à l'autre. On ne peut estimer leur rendement à partir de leur composition que pour un type de fromage déterminé produit dans des conditions très standardisées (outillage, technique). C'est ce qui a été fait par exemple pourle Cheddar aux U.S.A. (BABCock, Van StykE) et en Nouvelle-Zélande (Mc DowALI, I936) avec les résultats suivants :

$$
\begin{array}{ll}
\text { BABCOCK: } & R=2,5 \mathrm{C}+\mathrm{I}, \mathrm{IG} \\
\text { VAN SLYKKE \& PRICE: } & \mathrm{R}=\frac{(0,93 \mathrm{G}+\mathrm{C}-\mathrm{O}, \mathrm{I}) \mathrm{I}, 09}{\mathrm{I}, \mathrm{OO}-\mathrm{E}}
\end{array}
$$


Mc Dowali, :

$$
\begin{aligned}
R & =2,35 \mathrm{C}+\mathrm{I}, 07 \mathrm{G} \\
\mathrm{R} & =\mathrm{I}, 63 \mathrm{~A}+\mathrm{I}, 22 \mathrm{G} \\
\mathrm{R}^{\prime} & =\mathrm{I} .67 \mathrm{~A}+\mathrm{I}, \mathrm{I} 4 \mathrm{G} \\
\mathrm{R}^{\prime} & =2,42 \mathrm{C}+0,98 \mathrm{G}
\end{aligned}
$$

$\mathrm{R}$ : Poids de fromage frais sortant de presse obtenu à partir de roo $\mathrm{kg}$. de lait.

$\mathrm{R}^{\prime}$ : Poids de fromage âgé de $\mathrm{I} 4$ jours obtenu à partir de $100 \mathrm{~kg}$. de lait.

$G$ : Teneur du lait en matières grasses $\%$.

A : Teneur du lait en matières azotées totales $\%$.

$\mathrm{C}$ : Teneur du lait en caséine A.O.A.C. \%.

$\mathrm{E}$ : Humidité du fromage $\%$.

Dans ces conditions la seule base de référence commune pour caractériser la valeur fromagère des laits indépendamment du type de fromage fabriqué est la somme " caséine + matières grasses ", laquelle détermine le rendement en fromage sec.

Elle a cependant l'inconvénient d'accorder la même importance à la caséine et à la graisse, ce qui est faux du point de vue du rendement en fromage humide qui, seul, intéresse le producteur. Les formules précédentes avaient justement l'avantage de montrer le rôle prépondérant de la caséine et la nécessité d'attacher plus d'importance à celle-ci qu'au taux butyreux dans la sélection fromagère, à condition que ce ne soit pas au détriment de la qualité du fromage.

Qualité du fromage. - Du point de vue chimique on caractérise la qualité du fromage par la teneur en matières grasses $(\mathrm{G})$ de son extrait sec (S) ; par exemple, ce rapport $\mathrm{G} / \mathrm{S}$ doit être supérieur à $48 \mathrm{p}$. Ioo pour un gruyère de comté de bonne qualité (lait partiellement écrémé) et à 50 p. Ioo pour un Cheddar légal (lait entier). Or il varie en raison inverse du rapport Matières azotées Matières grasses du lait emprésuré, comme l'ont montré les travaux de Van SLyke (I894) sur le Cheddar dont nous pouvons retenir les repères suivants :

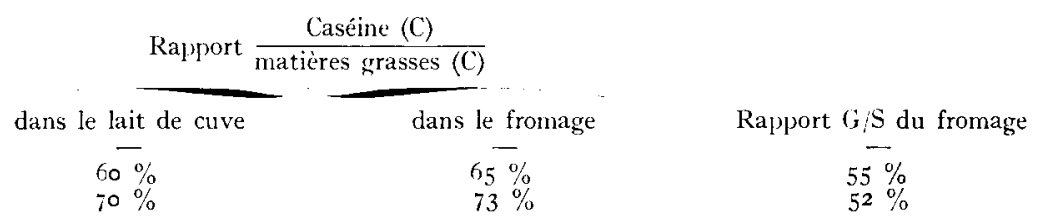

Mac Dowal, (I936) conclut qu'il ne faut pas dépasser un rapport $\mathrm{C} / \mathrm{G}$ de $72 \mathrm{p}$. Ioo dans le lait pour ne pas risquer de produire quelques fromages "maigres" (G/S inférieur à 50 p. I 000). De même G. Mocouor constate que pour obtenir des gruyères de Comté ayant un $\mathrm{G} / \mathrm{S}$ supérieur à 48 p. Ioo, il faut emprésurer des laits de cuve dont le rapport $\frac{\text { matières azotées totales }}{\text { matières grasses }}$ est inférieur à I03 p. Ioo ce qui correspond à un rapport $\mathrm{C} / \mathrm{G}$ d'environ 82 p. I 00 . 
Toutes nos vaches (tableau III, graph. 7) répondent à cette dernière condition et les Normandes présentent un très large excès de matières grasses (qui seraient d'ailleurs enlevées en partie par l'écrémage partiel dans certains types de fabrication); par contre un certain nombre de Frisonnes à taux butyreux faible ont un rapport $\mathrm{C} / \mathrm{G}$ trop élevé pour fabriquer des fromages type Cheddar suffisamment gras. Ces considérations très fragmentaires permettent de penser qu'après avoir éliminé ces vaches à lait pauvre on pourrait avant tout augmenter la teneur en caséine sans risquer de nuire à la qualité moyenne des fromages. (cf note ci dessous).

Coût énergétique. - Les variations relatives des teneurs en matières grasses et azotées doivent modifier le coût énergétique de la sécrétion de protéines car la valeur calorifique $(y)$ du lait dépend avant tout du taux butyreux $(x)$. A l'aide de la relation de Gaines et Overman (I938) : $y=304,8+$ II 4 , I $x$, nous avons estimé pour chaque vache (tableau III) la quantité de protéines (mg) produite par calorie de lait : elle varie de 43,7 à 49,0 chez les Frisonnes (moyenne 45,9) de 4r,o à 49,9 chez les Normandes (moyenne 45,6 ) et atteint un maximum de 52,3 chez notre unique Pie Rouge. En admettant que nos estimations de la valeur calorifique soient correctes et que le rendement énergétique de la production soit du même ordre chez nos différents animaux, nous pouvons penser que certaines vaches produisent les protéines à un coût énergétique plus faible : elles seraient d'autant plus intéressantes à rechercher que la quantité d'énergie consommée est le principal facteur limitant la production laitière.

En définitive ces hypothèses sur le rendement énergétique aboutissent aux mêmes conclusions pratiques que les études sur le rendement fromager : il faut augmenter avant tout la teneur en protéines (caséine) ainsi que le rapport matières azotées taux butyreux pour accroître le rendement du lait en fromage frais tout en diminuant le côtt énergétique de sa production; mais ceci à condition d'avoir un taux butyreux satisfaisant ( 35 grammes pour les laits de mélange) d’où la nécessité d'éliminer rapidement les vaches à lait pauvre en matières grasses (et, par là même, généralement en matières azotées).

De toute façon, il faut attendre les résultats de l'étude d'AurioL, et Mocguor sur les variations et la transmission héréditaire des aptitudes fromagères (héritabilité, progeny-test) des animaux Pie Rouge de 1'Est,

(I) Il est bon de signaler que, dans beaucoup) de régions, le rapport $G / S$ est susceptible de varier aver les circonstances économiques, la demande du consommateur... A certaines périodes, on préfère utiliser la graisse du lait sous forme de beurre et on fabrique des fromages "maigres ": parfois, au contraire, on fabrique des fromages, notamment certains types it pate molle, ayant une teneur en matières grasses tries élevée ( $G / S$ dépassant $60 \%$ par exemple). Haiz le problème de la sélection fromagère ne se pose que dans des régions où on fabrique un type de fromage bien déterminć, dont la composition ne varie qu'entre des limites étroites. 
pour savoir si la sélection fromagère, théoriquement possible, est économiquement réalisable.

\section{RÉSUMÉ}

I $^{0}$ Nous avons analysé les différentes liaisons entre les teneurs en matières azotées et en matières grasses de 855 échantillons de laits individuels prélevés au cours d'une lactation de 28 vaches, à raison d'un par semaine au cours des deux premiers et des deux derniers mois et d'un par quinzaine dans l'intervalle.

$2^{\circ}$ La première liaison apparaît au niveau intra-individuel pour l'ensemble des échantillons d'une même vache (tableau III) ; le coefficient de corrélation a été significativement positif pour I9 vaches ; sa valeur (de 0,06 à 0,92 ) traduit le degré de similitude dans l'évolution générale et dans les fluctuations passagères des deux teneurs au cours de la lactation, et dépend, avant tout, de la régularité du taux butyreux.

$3^{\circ}$ La corrélation interindividuelle (graph. 7) dans laquelle chaque vache est représentée par ses valeurs moyennes pondérées, a été hautement significative pour les $I 6$ Frisonnes $(r=0,8 I 6)$ mais non pour les II Normandes $\left(r=0,2 I_{3}\right)$; en moyenne la teneur en matières azotées augmente avec le taux butyreux, mais à un rythme beaucoup plus faible.

$4^{0}$ Ein raison de ces deux liaisons fondamentales à laquelle vient sans doute s'ajouter une liaison inter-races, on obtient toujours des corrélations positives pour les ensembles de laits individuels et, à fortiori, de laits de mélange ; leur valeur dépend avant tout de l'échantillonnage (graph. I) et ne permet aucune conclusion génétique.

$5^{\circ}$ Les résultats des analyses journalières des laits de 4 vaches, montrent que les variations journalières de la teneur en protéines sont 3 à 4 fois plus faibles que celles du taux butyreux (tableau V), et permettent de penser qu'il existe des différences individuelles dans la variabilité totale du taux butyreux d'une part,et dans la fraction de celle-ci qui est liée aux variations de la teneur en protéines d'autre part (graph. 6).

$6^{\circ}$ Grâce à la corrélation interindividuelle, la sélection classique sur le taux butyreux doit permettre une amélioration certaine de la teneur en protéines; celle-ci est lente par suite des variations du rapport matières 'azotées/matières grasses $(87$ à IoI, 3. chez les Frisonnes 73,3 à 96 , I chez les Normandes), mais elle est gratuite; il faut donc s'en contenter dans toutes les régions produisant d'abord des laits de consommation.

$7^{\circ}$ Par contre, pour augmenter efficacement la richesse en caséine des laits produits dans les régions strictement fromagères, il serait sou- 
haitable de faire une sélection directe en contrôlant systématiquement les variations individuelles de la teneur en matières azotées totales.

Reçu pour publication le I7 octobre 1956.

\section{REMERCIEMENTS}

Nous remercions M. Mocovot qui nous a guidés dans la discussion de la valeur fromagère des laits.

\section{RÉFÉRENCES BIBLIOGRAPHIQUES}

ANDERSEN (A. G.), LANGMACK (P. V.), (Ig23) - Beretn fra Forsogslab, no II3. Antoine (A.), (I952) - Bull. Inst. A gron. Gembloux, 20, 5 .

Auriol. (P.), (I956) - C. R. Acad. A gric., 42, 388.

Black (A.), I E Roy Voris (A.), (I934) - J. Agric. Res., 48, I025-Io32.

Bonnier (G.), Hansson (A.), Jarl (F.), (I946) - Acta Agric. Suecana, 2, (2), I59.

Bonnier (G.), Hansson (A.), (I946) - Acta Agriculturae Suecana II, I7I-I84. CoMberg (G.), (I95I) - Kühn Archiv., 64, 30I-64.

De Munter (P.), (I953) - Bull Inst. Agron. Gembloux, 21, (I-2) 29.

DE VUYST (A.), IMBEREChTS (R.), VerVACK (W.), (I953)-Zootechnia, 2, I37146.

DE VUYST (A.), IMBERECH'S (R.), (I955) - Revue d'A griculture, 8, 206.

Eyrard (A.) Hurel (C.) Boisseau (M.) (r945) C. R. Acad. Agric. France 32 I75. GaINes (W. L.), (I938) - J. Dairy Science, 8, 486-496.

GaInes (W. L.), OVERMAN (O. R.), (I938) - J. Dairy Sci., 21, 26I-27I.

GARTNER (R.), UIRICH (A.), (I95I) - Milchwissenschaft, 6, 8/9, 305-309.

GRABISCH (W.), (I953) - Züchtungskunde, 24, 3, I2I.

HANCOCK (J.), (r953) - N.Z.J.Sci.Tech., 35, 67.

Janse (L. C.), BACKhoven (L.), Roelofs (E. T.), (I954) - The Netherlands milk and dairy journal, 8, 39-40.

Janse (L. C.), Bakmoven (H. G. A.), Roelofs (E. T.), (r954) - Bulletin HerdBook F. F. P. N., 34, 39 .

JARRIGE (R.), RosSE'TTI (C.), (I957 a) - Annales Zootechnie, 6, $4 \mathrm{I}$.

JARRIGE (R.), ROSSETTI (C.), (I957 b) - Annales Zootechnie, 6, 64.

KAY H. D. (I955) Agriculture 62369.

Kahlenderg (O. J.), Le Roy Voris (A.), (I93I) - J. Agric. Res., 43, 749-755.

KRIZENECKY (J.), (I943) - Ztschr. Tierzüchtung Züchtungsbiol., 55, IOI.

Larsson (E. L.), Platon (J. B.), Thome (K. E.), Hansson (A.), (I949) - Congrès $\left(\mathrm{I} 2^{\mathrm{e}}\right)$ Inter. Laiterie, Stockholm, Vol. 1, 377-85.

LARSSON (E. L.), (I950) - Mejeritek. Meddeland., 11, I4.

Lonka (T.), (I947) - Maataloust Aikakausk, 19, 8-2I.

Lonka (T.), (I952) - Maataloust Aikakausk, 24, (4), I76-8r.

MCDowali (F. H.), (I936) - Dairy Res. Inst. (N. Z.) Pub. no gr.

Mocouot (G.). - Résultats non publiés.

Mork (R.), (I942) - Meld., ñ 21, Norges Landbruk Meieri.

Musgrave (S. D.), Salisbury (G. W.), (I952) - J. Dairy. Sci., 35, 2, I74-78.

Nesent (R.), Gruhn (R.), Freimuth (V.), (I95I) - Arch. für Tiernahrung, 2, I $52-165$.

NESENI (R.), Korprich (H.), (I947) - Milchwissenschatt, 7, 328.

Overman (O. R.), Sanmann (F. P.), Wright (K. E.), (Ig29) - Illinois Agric. Exp. Sta. Bull., 325.

Overman (O. R.), Garre'T' (O. F.), Wright (K. E.), Sanmann (F. P.), (I939) - Illinois Agr. Sta. Bull., 45\%. 
Overman (O. R.), Kevis (R. J.), Craine (E. M.), (I953) - Illinois A gric. Exp. Sta. Bull., 56\%.

PIEL (H.), (I953) - Züchtungskunde, 24, (6), 266-79.

Platon (B.), Sjostrom (G.), (I940) - Cités par Larsson (I950).

Poijarvi (I.), Listo (E. M.), (I930) - Cités par Lonka (I947).

PRESSLER (H.), (I937) - Dissertation Bonn.

Provan (A. L.) (I955) J. Soc. Dairy Tech. 856.

Rowland (S. J,) (I946) Dairy Industries II 656.

SchmidT (J.), (I943) - Züchtungskunde, 17, 303.

Stuber (O.), Gobeit (J.), (I952) - Milchwiss. Berichte, Wolfpassing, 1, I-33.

STUbeR (O.), (I953) - XIII Congrès Inter. Laiterie, II, 30 I.

Timpe (H.), (I899) - Cités par McDowal. (I936).

VALEN (I.), (I953) - XIII Congrès Inter. Laiterie, Vol. II, 296.

VANSChOUbrotck (F.), WiliteMs (A. E. R.), (I955) - Zootechnia, 4, (2), 76-88. VAN Slyke (L. L.), (Igo8) - J. Amer. Chem. Soc., 30, ri6-II86.

VAn Slyke (L. L.), Price (W. V.), (I952) - Cheese Orange Judd. Pub. Co.

Vessereau (A.), (I948) - Méthodes statistiques en biologie et en agronomie, (Baillière).

\section{ADDENDUM}

Le récent travail de $A$. Robertson, R. Waite et J. C. D. White (I956) sur 500 couples mère-fille Ayrshire vient d'apporter la première estimation de la corrélation génétique entre le taux butyreux et les teneurs en matières azotées totales $(0,48)$ et en caséine $(0,56)$. Ces valeurs sont légèrement supérieures aux corrélations phénotypiques inter-individuelles, qui ont été respectivement chez les mères et chez les filles de 0,372 et $0,4 \mathrm{I} 6$ pour les matières azotées totales, 0,428 et 0,43 I pour la caséine, (J. Dairy Res. 23, 65-82). 